\title{
Déplacements de consommation et transferts de richesses en Île-de-France
}

\author{
Jean-François Ruault*, Laurent Proulhac \\ *Université Paris-Est, Laboratoire Ville Mobilité Transports (LVMT) \\ UMR T-9403 ENPC IFSTTAR UPEMLV
}

Bât. Bienvenue, 6 et 8 avenue Blaise Pascal, 77455 Marne-la-Vallée cedex 2

\section{Résumé}

L'importance des transferts de richesses dans la construction économique des territoires ne cesse d'être soulignée. Si un premier mouvement est observable des lieux d'emploi aux lieux de résidence, un second tient à la dissociation entre espaces de consommation et espaces résidentiels. Cet article propose une mesure inédite des transferts interdépartementaux de richesses en Île-de-France par le prisme des dépenses de consommation. Il s'appuie sur le croisement d'une enquête de la mobilité quotidienne des Franciliens et d'une autre relative à leurs dépenses. L'objectif est ici d'améliorer la compréhension de la performance économique des territoires, de mesurer l'attractivité commerciale et ses effets sur la redistribution géographique des richesses. Les résultats obtenus soulignent des déséquilibres structurels et l'éclatement des lieux de consommation, entre ancrage parisien des activités de spectacles et de loisirs, ainsi que la restauration, et une spécialisation de la périphérie autour des achats courants et exceptionnels. L'analyse des transferts de richesses interdépartementaux expose par ailleurs la force des interdépendances qui participent de la structuration de la métropole.

Mots clefs : mobilité, sciences régionales, économie territoriale, développement local, économie résidentielle, économie présentielle, consommation, commerce, métropolisation, Paris, Île-de-France.

Adresse email : jean-francois.ruault@enpc.fr doi :10.3166/ges.16.91-122 ㄷ 2014 Lavoisier, Paris. Tous droits réservés. 


\begin{abstract}
Summary
Consumer trips and money flows in the Paris Region. Transfers of wealth are increasingly shown as a key component of the community economic development. It is well-known that commuters contribute to moving wealth from their workplaces to the residential areas where they live. Moreover a second wave also exists from residential areas to spaces for consumption. The objective of this paper is to propose an original measure of the subregional money flows related to consumer spending in the Paris Region. This requires a cross data operation analysis using a regional travel as well as a national household budget survey. The purpose is to improve the regional economics understanding by measuring consumer attraction and wealth redistribution. The results highlight structural imbalances and the spaces for consumption spreading. Leisure and entertainment are concentrated in Paris when the suburbs are specialized in providing manufactured goods. These subregionals transfers of wealth show the interlinkages shaping the Paris metropolitan area.
\end{abstract}

Keywords: mobility, shopping trips, regional science, urban economics, economic development, economic base, consumption, retail, Paris.

(C) 2014 Lavoisier, Paris. Tous droits réservés.

\title{
Introduction
}

S'appuyant sur de nombreux cas régionaux, C.C. Williams a alerté les économistes régionaux à propos de la force motrice que peut revêtir la consommation pour le développement local (Williams, 1997). S'inquiéter de savoir où les consommateurs dépensent leurs revenus n'est pas une préoccupation marginale pour l'économie des territoires. Le commerce constitue d'ailleurs un sujet extrêmement sensible chez un grand nombre d'élus locaux : lutte contre le retrait de l'offre globale de services pour les territoires ruraux, préservation des densités commerciales face à la concurrence périphérique pour les centres urbains, souci de l'emploi local et du ménagement de la ressource foncière pour les espaces périurbains ${ }^{1}$ (Constans-Martigny et Ruault, 2012). Ces inquiétudes traduisent pleinement la tension qui frappe les territoires à l'heure où toute captation de dépenses compte pour animer et sauvegarder l'activité économique locale.

Si le commerce a toujours été une fonction structurante de la ville (Beaujeu-Garnier et Chabot, 1963; Berry, 1971; Desse, 2001; Péron, 2004; Capon, 2011; Delaporte, 2012), au point parfois d'être le principal moteur de développement (Weber, 1921), l'évolution des mobilités rend aujourd'hui cette fonction économique plus disputée et éclatée qu'autrefois. Au point que les aménités de consommation confèrent un avantage stratégique aux grandes villes inscrites dans une compétition mondiale (Glaeser et al., 2001 ; Rappaport, 2008). A fortiori, comprendre et mesurer l'importance des transferts de richesses entre territoires s'affirme comme une dimension structurante de l'analyse économique locale.

Pourtant, comme le souligne Laurent Davezies (2007), peu de travaux se sont penchés sur la redistribution des richesses induites par la consommation des ménages. Devant ce manque, le présent article propose une démarche exploratoire permettant de saisir l'importance de ces transferts de richesses, mesurés en dépenses monétaires à l'échelle de l'île-de-France.

\footnotetext{
${ }^{1}$ Ici, comme dans la suite de l'article, le périurbain désigne l'espace, dissociable morphologiquement de la densité et de l'unité bâti de l'agglomération, tout en étant fortement sous son influence.
} 
La région francilienne s'inscrit dans un mouvement de déconcentration des populations, des emplois (Halbert, 2004) et des activités commerciales et récréatives (Desse, 2001; Delaporte et Courel, 2006; Mérenne-Schoumaker, 2008), favorisant ainsi l'émergence de nouvelles centralités commerciales périphériques (Delage, 2011). La dynamique actuelle de réorganisation du commerce au profit de la périphérie bouleverse le paysage sans remettre fondamentalement en cause le rôle structurant de la centralité parisienne (Fleury et al., 2012, Delage, 2011). Paris reste à bien des égards le centre de consommation de référence, de par son héritage historique, son cosmopolitisme et son rayonnement international (Glaeser et al., 2001). Dans le même temps, et en lien avec ces dynamiques spatiales, les comportements de mobilité quotidienne des individus se sont modifiés et complexifiés (Massot et Orfeuil, 2005). Ces tendances de longue date ont affecté les pratiques de consommations des ménages (Desse, 2000 ; Gasnier, 2007).

Dans ce cadre, il nous apparaît nécessaire d'interroger les transferts de richesses induits par les dépenses de consommation en Île-de-France. Trois principaux objectifs sont ainsi visés : (1) mesurer la performance économique des territoires d'une région métropolitaine à l'aune des lieux de valorisation des richesses et non seulement des lieux de leur création ou de leur détention; (2) déterminer quels sont les territoires attractifs auprès des consommateurs franciliens; et (3) esquisser une géographie des flux de richesses approchée comme un révélateur de liens économiques et d'interdépendances entre différents territoires d'une même métropole.

Pour répondre à ces questions, nous exposons, dans une première partie, le cadre théorique et les éléments de contexte. Le papier détaille ensuite la méthodologie retenue et présente dans une troisième partie les résultats.

\section{Quand détenir des richesses ne rime pas avec valoriser ces richesses}

La dernière décennie a été marquée par une recrudescence de travaux revisitant la théorie de la base économique et conférant aux pratiques spatiales des ménages, et à l'économie résidentielle en particulier, un rôle déterminant dans la redistribution des richesses. L'effet multiplicateur des revenus basiques reste néanmoins incertain face à des pratiques de consommation devenues plus mobiles. Dans ce cadre, le cas de la métropole francilienne est particulièrement séduisant de par la variété et l'étendue des espaces de vie qu'elle abrite.

\subsection{Valoriser les richesses détenues localement}

La théorie de la base se prête particulièrement bien à l'appréhension des effets économiques de la consommation locale. Dans son plus simple appareil (figure 1, voir page suivante), elle adosse la dynamique de développement régional à la stimulation de l'activité économique par la mise en circulation de richesses issues des flux monétaires interrégionaux.

Ces richesses, ou revenus basiques, peuvent trouver leur origine de diverses sources : commerce interrégional, attraction résidentielle, aides sociales, etc. Une fois dépensés sur le marché régional, les revenus basiques ont un effet multiplicateur, et participent à la création d'emplois et à la formation de nouveaux revenus. Ces emplois et revenus induits 
sont dits «non-basiques », « domestiques » ou encore « présentiels ${ }^{2} »$. De cette posture, tout changement majeur de l'économie locale passe par un changement de la base.

Figure 1 : Schéma de la théorie de la base économique

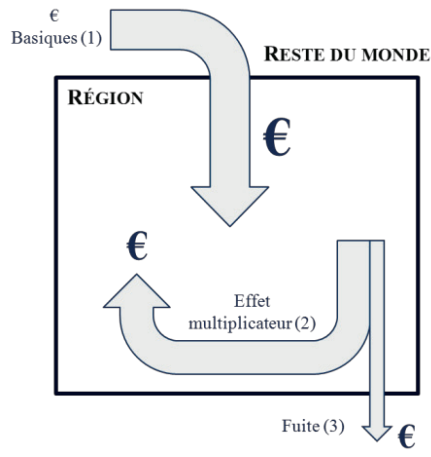

\subsubsection{La base résidentielle en réponse à une théorie décharnée}

Si la théorie de la base constitue un cadre de réflexion majeur de l'économie régionale, il n'existe pourtant aucun modèle ni outil d'analyse stabilisé pour lui donner corps (Krikelas, 1992; Gonnard, 2001). L'approche par les intrants monétaires est en effet rendue très difficile d'application par le caractère épars et incomplet des données sur les revenus ${ }^{3}$, leurs sources et leur distribution géographique. Une difficulté encore plus prononcée lorsqu'il s'agit d'étudier les chiffres d'affaires ${ }^{4}$ ou les dépenses de consommation des ménages ${ }^{5}$. En l'absence d'une comptabilité détaillée des flux monétaires interrégionaux, les méthodes d'analyse par l'emploi font école. Or ces méthodes reposent sur le partitionnement, normatif et mouvant, des activités économiques selon qu'elles exportent ou qu'elles servent le marché local et ont introduit une digression théorique où l'exportation serait la principale source de revenus basiques. Pour D.H. Farness (1989), cette théorie de la base exportatrice souffre de problèmes méthodologiques majeurs dans la mesure où le revenu dérive de plus en plus de transferts de richesses de visiteurs non-résidents et d'autres ressources externes. En outre, la « déterritorialisation » progressive du commerce et de la consommation ne garantissent plus mécaniquement d'effet multiplicateur aux territoires productifs et remettent en cause la fonction motrice de la production à l'échelle locale (Madry, 2012). La théorie de la

${ }^{2}$ L'Insee s'est doté d'une nomenclature distinguant les activités présentielles des activités non-présentielles. Les activités présentielles correspondent aux activités constitutives d'une offre locale de biens et de services à destination des personnes présentes localement (résidents, touristes, excursionnistes...). À l'inverse, lorsque l'essentiel du marché d'une activité se situe en dehors de l'espace local alors celle-ci est réputée non-présentielle.

${ }^{3}$ Les revenus sont ici entendus comme toutes les sommes perçues par les ménages et alimentant leur pouvoir d'achat. Ils ne se limitent donc pas aux revenus du travail mais tiennent également aux revenus du capital ou encore aux aides sociales. T. Piketty montre combien il est difficile de collecter ces informations à l'échelle même des nations (Piketty, 2013).

${ }^{4}$ Un exemple emblématique est l'enquête Fortune auprès des entreprises d'Oskaloosa (1938) qui permettait d'établir le montant des recettes et des dépenses extérieures. La réalisation de l'enquête fut particulièrement délicate.

${ }^{5}$ Ce sont aujourd'hui principalement les Chambres de Commerce et d'Industrie et certains bureaux d'études spécialisés qui établissent, à l'échelle de départements ou d'agglomérations, des estimations des dépenses locales. 
base exportatrice, et par amalgame la théorie générale, se sont ainsi vues rapidement critiquées au point d'être discréditées (Gonnard, 2001).

Depuis une dizaine d'années néanmoins, des auteurs revisitent les fondements historiques $^{6}$ de cette théorie et cherchent à en renouveler l'interprétation (Talandier, 2007; Davezies, 2008; Dissart et Vollet, 2010). Ils se démarquent par leur détachement de la théorie exportatrice, leur approfondissement des sources de revenus basiques et par le réinvestissement des approches par l'emploi pour l'analyse du seul effet multiplicateur. L. Davezies a notamment élargi la base économique à quatre grandes sphères de revenus : la base productive privée (traitements et salaires de la base exportatrice), la base résidentielle (salaire des migrants-alternants, pensions de retraite, dépenses touristiques...), la base publique (salaires des fonctionnaires) et la base sociale (allocations et aides sociales). La base résidentielle se révèle ainsi être la première source ${ }^{7}$ de revenus basiques pour les territoires, aussi bien dans les zones d'emploi que dans les aires urbaines françaises (Davezies, 2008). Un constat qui alimente aujourd'hui un nombre grandissant de travaux académiques et professionnels sur l'économie résidentielle ou présentielle ${ }^{8}$. Cet engouement pour la composante résidentielle ne doit cependant pas éclipser le fait que ces travaux ont profondément contribué à réhabiliter la théorie de la base et à mettre plus globalement en lumière la puissance des mécanismes redistributifs, publics ou privés, dans l'économie des territoires.

\subsubsection{L'éclosion d'une théorie de la base de consommation ${ }^{9}$}

Une autre déclinaison de la théorie de la base, présentée comme une alternative à sa variante exportatrice, est la théorie de la base de consommation. Elle suggère qu'en réduisant les flux monétaires sortants, une région accroît son activité et assure sa croissance. Autrement dit, cette approche s'intéresse au bas du modèle circulatoire de la base économique. Celle-ci alimente régulièrement les discours politiques nationaux ou subnationaux sous la forme d'un appel au «produire et consommer localement ». Il faut bien comprendre que dans les économies développées, les gisements de croissance tendent en effet à se tarir (Davezies, 2010), au point que la consommation des ménages en est souvent devenue la bouée de sauvetage ${ }^{10}$. Pour soutenir la croissance, il est donc devenu stratégique de soutenir la consommation, quitte à souffrir parfois d'excès dont le surendettement est le symbole (Frank, 2000; Moati, 2012). Le souci de la vulnérabilité et de la soutenabilité du développement territorial a ainsi conduit certains décideurs locaux à promouvoir les circuits courts et l'achat local (Kwon, 2010).

${ }^{6}$ Selon G. Krumme cité par S. Gonnard (2001), la paternité de la théorie de la base reviendrait à l'économiste allemand Werner Sombart (1916) bien que l'esprit de la théorie se retrouve dans les travaux de Richard Cantillon (1725) ou de Pieter de la Court (1659).

${ }^{7}$ Ce qui met à mal la théorie de la base exportatrice.

${ }^{8}$ L'économie présentielle, qui s'intéresse aux retombées locales permises par la présence temporaire ou durable d'individus en un territoire, est un concept qui tend progressivement à se substituer à celui d'économie résidentielle sémantiquement plus restreint. Dans leur usage académique, il n'y a pas toujours de différences mais l'ambiguïté entraîne des confusions et quelques rabattements de l'économie résidentielle à la seule contribution des habitants.

${ }^{9}$ Il s'agit du concept Anglo-Saxon de « consumption base », traduit ici par «base de consommation » mais aussi abordé en termes de «base consommatoire » par Pierre Veltz (2012) ou de «base consommatrice » par Magali Talandier (2013).

${ }^{10}$ La consommation des ménages compte aujourd'hui pour pas moins de deux tiers du PIB français. 
La substitution aux importations aurait, selon G.A. Erickcek et B.R. Watts (2007), potentiellement le même impact qu'un accroissement de l'exportation. De fait, dès lors que l'argent dépensé est « nouveau » pour le territoire, il génère un accroissement net de l'activité régionale. Lorsqu'une région éprouve des difficultés à créer ou capter des revenus basiques, stimuler l'activité par la demande locale peut alors se révéler salvateur. C'est pourquoi les régions rurales, les franges périurbaines, les petites villes voire certains quartiers déprimés ont tant fait l'objet de stratégies de revitalisation économique par la régénération du tissu commercial. Plus encore, la base de consommation a montré sa capacité à conduire des expérimentations et des innovations locales susceptibles de s'exporter par la suite (Jacobs, 2000; Cortright, 2002). A. Markusen (2007; 2009) considère à ce titre qu'en agissant sur les structures de consommation, il est possible d'améliorer la rencontre entre l'offre et la demande locale. Il suffit alors de substituer des produits et services locaux aux importations des ménages de la région, ou encore d'éveiller la demande latente en rendant accessible une offre qui ne l'est pas encore.

De toute évidence, s'emparer de dépenses de consommation volatiles est porteur d'enjeux majeurs pour le développement des territoires (Talandier, 2013; Talandier, Jousseaume, 2013).

\subsection{Les déplacements les plus ordinaires façonnent l'économie présentielle}

Lorsqu'il s'agit d'apprécier l'importance des transferts induits par les dépenses de consommation, la littérature scientifique s'intéresse principalement au cas des dépenses touristiques. Des travaux en France (Vollet, 1998; Lejoux, 2002; Terrier, 2007; Lapeyronnie, 2009; Vacher et Vye, 2011) et à l'étranger (Fletcher, 1989; Archer, 1996; Stynes, 1997; Greiner et al, 2004; De la Mata et Llano-Verduras, 2011) ont ainsi participé à la reconnaissance du tourisme en tant que source motrice du développement local. Pour autant, il convient de ne pas négliger les dépenses plus ordinaires. Faire les courses, assister à une pièce de théâtre ou aller se restaurer sont autant de pratiques partagées participant localement à d'importants transferts de richesses.

\subsubsection{La dimension structurante des déplacements de consommation}

L'individu est gouverné par une hiérarchie de besoins allant des plus élémentaires (manger, dormir, boire...) aux plus complexes (s'identifier, créer...) (Maslow, 1943). Il se construit ainsi autour d'activités qui lui prennent du temps et nécessitent très souvent une mise en mouvement. Consommer constitue l'une de ces activités. S'il est possible de consommer sans se déplacer, il faut noter que l'essentiel de l'offre commerciale et récréative s'est construite autour de l'icône d'une classe moyenne mobile (Moati, 2012). Dans ces conditions, la consommation est devenue une activité structurante de la mobilité quotidienne des individus. En 2008, les motifs « achats » et « loisirs » représentaient respectivement $12 \%$ et $8 \%$ des déplacements hebdomadaires observés en France. Si l'on exclut les retours au domicile, qui gonflent considérablement le nombre de déplacements, ces deux motifs représentent le tiers de la mobilité quotidienne ${ }^{11}$ et même davantage le week-end ${ }^{12}$.

\footnotetext{
${ }^{11}$ Précisément $20 \%$ pour les « achats » et $13 \%$ pour les « loisirs ».

${ }^{12}$ Le week-end, $57 \%$ des déplacements sont effectués pour des motifs « achats » ou « loisirs ».
} 
Ainsi, aujourd'hui, dans la norme, le consommateur doit être mobile ${ }^{13}$. La capacité à se déplacer constitue non seulement une ressource mais aussi une exigence (Massot, 2010). Pour autant, cette capacité à être mobile n'est pas la même selon les individus et se traduit par d'importantes inégalités qui fracturent la société (Le Breton, 2005; Orfeuil, 2010). En 2010, les Franciliens les plus riches ont par exemple effectué 1,6 fois plus de déplacements pour des motifs «achats » ou « loisirs » que les plus pauvres. De même, les distances parcourues au quotidien pour ces deux motifs sont multipliées par 1,9. Cette inégale capacité des individus à se mouvoir produit donc des pratiques de consommation spécifiques et façonne des bassins de consommation d'amplitude variable. En d'autres termes, la plus grande mobilité des sociétés contemporaines doit être entendue comme un attribut dominant et non comme une réalité partagée.

La délimitation de zones de chalandises par les distributeurs est devenue très souvent une illusion face à l'émergence de comportements de consommation complexes. L'idée que l'activité commerciale est soutenue par une clientèle polaire ${ }^{14} \mathrm{~s}^{\prime}$ effrite à l'avantage d'un intérêt croissant pour la clientèle de passage (Michaud-Trevinal et Cliquet, 2002; Madry, 2012). Dès lors, la question se pose au sein des métropoles de savoir si la clientèle de passage n'a pas pris le pas sur la clientèle polaire.

\subsubsection{La société hyper-mobile en toile de fond}

L'avènement d'une société de consommateurs mobiles (Ascher, 2005) conduit à la redéfinition du lointain et du proche (Péron, 2001). La métrique de l'accès se substitue à celle de la proximité et redéfinit ainsi les stratégies de localisation des équipements commerciaux. "Toute la hiérarchie commerciale traditionnelle, fondée sur la proximité et l'éloignement géographique, devient caduque »(Van de Walle, 2005). Il s'agit aujourd'hui pour les distributeurs de privilégier l'accessibilité, d'abord routière, en ciblant les principaux nœuds d'échange, et plus généralement autour des lieux de transit, comme les gares ou les aéroports. Au-delà des lieux de transit, les distributeurs cherchent de plus en plus à accompagner le consommateur au quotidien, via le développement du $\mathrm{m}$-commerce ${ }^{15}$, ainsi que par la conception d'une offre dont le dimensionnement et l'agrément sont en mesure de contenir le client mobile et de le rendre captif pour la journée ou la demi-journée (Michaud-Trevinal et Cliquet, 2002).

L'éclatement spatial des activités dans les villes, permis par le développement automobile, étend l'éventail de l'offre de consommation rapidement accessible et favorise l'élargissement des territoires de vie. Cette logique est d'autant plus puissante que la pénibilité de la mobilité urbaine incite à une plus ferme rationalisation des déplacements, et en particulier au recours au chaînage des déplacements. Ces chaînages moins normés, plus complexes et diversifiés, ont donné naissance au concept de «pérégrination» (Rollier et Wiel, 1993).

\subsubsection{Des changements propres aux comportements de consommation}

Avec l'apparition d'une économie fordienne, organisée autour d'industries de production de masse, s'est développé un nouvel intermédiaire indispensable à la mise sur le marché

\footnotetext{
${ }^{13} \mathrm{Au}$ sens physique et virtuel (l'e-commerce par exemple) du terme.

${ }^{14}$ La clientèle est polaire lorsqu'elle est captive de l'offre commerciale de proximité, cette proximité étant établie par rapport à un pôle commercial et au regard du lieu de résidence.

${ }^{15}$ Le m-commerce regroupe l'ensemble des transactions effectuées depuis un téléphone mobile.
} 
des biens et services produits : la grande distribution (Moati, 2011). Ces changements apparaissent dans un contexte d'essor économique sans précédent et se traduisent par une élévation considérable des niveaux de vie. Les biens les plus inaccessibles, tels que la propriété immobilière ou l'automobile, deviennent à la portée du très grand nombre. Les aspirations individuelles grandissent et les modes de vie changent. L'organisation du marché des biens et services, qui lie une population à des lieux de consommation, est bouleversée par l'apparition de nouvelles centralités commerciales, de nouveaux rapports de hiérarchie, qui débordent de la figure de l'agglomération et se diffusent dans un urbain élargi (Berry, 1971; Beaujeu-Garnier et Delobez; 1977). Une révolution commerciale telle qu'elle soulève les plus vives inquiétudes quant au devenir de la fonction commerciale des centres-villes et de leur capacité à se moderniser (Metton, 1984).

Dans ce contexte, les distances parcourues ont augmenté. Satisfaire ses besoins de première nécessité est aujourd'hui vécu comme une corvée, un temps contraint à rationnaliser (Bondue, 2004). L'accès à l'automobile et à la réfrigération a notamment permis au consommateur de pratiquer le regroupement des achats et de réduire sa fréquence hebdomadaire d'approvisionnement de 4,4 en 1988 à 3,2 en 1995 (Babayou et Volatier, 1998). Par rebond, les petits commerces de proximité ont vu leur fréquentation se réduire à l'avantage des grandes surfaces plus propices à l'achat diversifié et en gros volumes. Par ailleurs, d'autres types d'achat, à mi-chemin entre l'achat contraint et l'achat récréatif (équipement de la maison, habillement...) sont constitutifs d'une offre rare et polarisée à l'échelle métropolitaine nécessitant de parcourir des distances plus importantes. Enfin les activités récréatives et de loisirs répondent à leur propre logique et peuvent motiver des déplacements particulièrement longs s'affranchissant des périmètres régionaux ${ }^{16}$.

\subsubsection{Des revenus basiques vulnérables aux pratiques de consommation}

Les comportements actuels de mobilité questionnent l'organisation des espaces de l'action publique, des périmètres institutionnels dont les individus s'affranchissent au quotidien. Les territoires sont effectivement des espaces passants, autant que des espaces résidentiels, où il n'est plus possible de raisonner à l'aune des seuls gouvernements locaux (Béhar et al., 2009). Cette plus grande mobilité du consommateur induit de surcroît une plus grande incertitude pour les territoires quant à leur capacité à valoriser leurs revenus basiques en dépenses locales et à ainsi assurer un effet multiplicateur en termes d'emplois et de revenus. De fait, le consommateur n'est pas toujours pleinement en mesure de satisfaire ses besoins à l'endroit où il réside et il est alors libre d'aller dépenser son argent où bon lui semble (Tiebout, 1956) ${ }^{17}$. A fortiori, de nombreux établissements ne s'appuient pas directement sur la population résidente pour exercer leur activité (Wesley et LeHew, 2002; Bowles, 2007). De par leur dimensionnement ou leur rareté, ou bien encore parce qu'ils s'implantent dans les lieux passants, ces équipements ciblent pour tout ou partie une clientèle de passage. C'est le cas en particulier des équipements touristiques, des hôpitaux et cliniques, des parcs d'attractions, des grands centres commerciaux, de l'essentiel de la restauration rapide ou encore du commerce de gare et d'aéroport.

\footnotetext{
${ }^{16}$ Le parc Eurodisney à Marne-la-Vallée en est un exemple particulièrement emblématique avec une zone d'influence s'étendant à toute la France et une partie de l'Europe.

${ }^{17}$ Charles Tiebout parle de « vote par les pieds » ou l'aptitude des individus à se mouvoir et se rendre où bon leur semble afin de maximiser leur utilité.
} 
Par ailleurs, au cours des années 2000, la production de surfaces commerciales est entrée dans une bulle spéculative et a cru bien plus vite que la consommation des ménages (Madry, 2011). Autrement dit, le dimensionnement de l'offre ne s'est pas ajusté à la demande mais s'est construit indépendamment. Si des mécanismes autorégulateurs sont à l'œuvre, ils sont de toute évidence défaillants et il n'est plus permis de tenir le commerce pour strictement captif du marché local. Il y a donc une certaine vulnérabilité de l'économie locale dès lors que s'affirment des dynamiques d'évasion des consommations, qu'elles soient permises par la mobilité quotidienne des ménages, l'insuffisance de l'offre locale ou l'existence d'un contexte concurrentiel.

\subsection{L'île-de-France, cadre d'étude de la consommation métropolitaine}

L'Île-de-France est une région pionnière en matière de concepts commerciaux. Elle a été la première en France à compter des grands magasins, des magasins populaires, des hypermarchés, des centres commerciaux, des hard-discounters ou encore des zones d'activités commerciales (Delaporte, 2012). Cela fait de l'Île-de-France un cadre d'étude particulièrement intéressant.

\subsubsection{La volatilité du consommateur francilien}

Le caractère pionnier de la région est favorisé par son contexte métropolitain et des décennies d'interventionnisme régional en matière de logement et de transport. Au quotidien, le consommateur francilien se déplace aujourd'hui un peu plus vite qu'hier, parcourt de plus grandes distances, structure ses déplacements autour d'une plus grande variété de modes de transport et inscrit ses pratiques au sein de zones de chalandises plus floues. En outre, lorsqu'il s'agit d'identifier les contours d'un système productivo-résidentiel francilien, c'est-à-dire d'un espace « intégrant les différentes fonctions territoriales (production, logement, loisirs, retraite...) » (Talandier, 2012), celui-ci s'étend à toute la France. De fait, le Francilien est celui qui passe le plus de temps en dehors de sa région de résidence et participe ainsi d'un non négligeable manque à gagner pour la région (Terrier, 2007). Ce déficit de consommation, observé en Île-de-France (Davezies, 2007), tient également à un tassement du pouvoir d'achat. La mobilité francilienne demeure néanmoins une clé de lecture privilégiée qu'il convient d'explorer.

\subsubsection{Paris, le commerce distractif}

À l'instar des grandes métropoles, l'offre commerciale francilienne est riche et diversifiée. Paris y concentre une large part de l'activité, à la fois par l'inertie de la concentration commerciale historique mais également par l'attraction exceptionnelle qu'elle exerce. Ainsi, les grands magasins (Printemps-Hausmann, Galeries Lafayette, BHV ...), les musées nationaux (Le Louvre, Orsay, Cité des Sciences...) ou encore l'incroyable concentration des salles de spectacles témoignent du rayonnement métropolitain dont jouit Paris (Padeiro et al., 2014).

Néanmoins, il convient de noter que les fonctions commerciales et récréatives ne sont pas uniformément distribuées dans le tissu urbain. De fortes centralités sont identifiables aux abords des sites touristiques, des grandes artères (rue de Rivoli, boulevard Hausmann, boulevard Saint-Michel...), de certaines places (place Vendôme, place d'Italie, place des 
Ternes...) ou encore autour des gares (Delage, 2011). La hausse des loyers commerciaux observée depuis plusieurs décennies s'est également traduite par le recul du commerce indépendant et la migration vers la banlieue ${ }^{18}$ d'un grand nombre d'activités à faible profit ou nécessitant de grandes surfaces d'entreposage. Le foncier ainsi libéré a profité au commerce franchisé, aux supérettes alimentaires, mais plus encore à une foisonnante offre de services à la personne ${ }^{19}$. L'attractivité commerciale de Paris semble aujourd'hui se spécialiser autour de l'expérience urbaine, de la rareté et de l'usage.

\subsubsection{Une complémentarité centre-banlieue}

La révolution automobile amorcée dans les années soixante a favorisé l'émergence d'une nouvelle offre commerciale en périphérie et autorisé l'expérimentation de concepts commerciaux spacivores en dehors du tissu urbain dense. La banlieue d'abord puis le périurbain plus récemment, ont pu soulager les distributeurs de la pression foncière parisienne, en proposant des sites accessibles et visibles pour s'y implanter (Mangin, 2004). L'apparente mise en concurrence se présente davantage, pour l'offre commerciale de banlieue du moins, comme un complément majeur à une offre parisienne de plus en plus spécialisée. Au cours de la dernière décennie, on observe en effet que beaucoup d'activités commerciales et des services en déclin à Paris progressent en banlieue (Ruault, 2012). Il convient cependant de distinguer la proche banlieue de la banlieue éloignée. Cette dernière, d'intégration plus récente à l'agglomération, a bénéficié d'une première vague de «ceinturation » commerciale de Paris autour de l'A86 et justifie maintenant d'un niveau d'équipement conséquent (Val d'Europe à Chessy, Trois Fontaines à Cergy, le projet d'Europa City à Gonesse...). À l'inverse, l'offre commerciale de proche banlieue se trouve aujourd'hui atrophiée entre Paris et les centres commerciaux de la banlieue éloignée.

\subsubsection{Les métamorphoses du périurbain}

En dépit de son explosion démographique, l'offre commerciale périurbaine reste encore aujourd'hui sous-dimensionnée et peu diversifiée. Ce retard est cependant l'objet d'un fulgurant effet de rattrapage. Entre 1998 et 2008, l'emploi domestique ${ }^{20}$ a progressé de $15 \%$ en moyenne au sein de l'aire urbaine parisienne et de $23 \%$ pour le seul périurbain (Ruault, 2012). Ce taux de croissance est supérieur à celui de Paris $(+14 \%)$ et à celui de la banlieue éloignée $(+18 \%)$. Ce rattrapage s'est construit sur la base d'une seconde vague de «ceinturation » commerciale qui s'est étendue jusqu'en grande couronne, notamment aux abords de la voie express Francilienne, et qui aujourd'hui se voit rattrapée par l'expansion de l'agglomération parisienne ${ }^{21}$. Une offre qui se développe par substitution d'achats précédemment effectués dans l'agglomération par les périurbains. Longtemps incapable d'absorber l'accroissement de la demande locale, le périurbain semble aujourd'hui refaire une partie de son retard.

\footnotetext{
${ }^{18}$ Dans l'article, l'usage du mot «banlieue » se limitera strictement à sa définition géographique en tant que continuité bâtie d'un centre urbain au-delà de ses propres limites administratives.

${ }^{19}$ Bars à sourire, restauration rapide...

${ }^{20}$ Pour rappel, les emplois domestiques d'un territoire sont les emplois des activités locales soutenues par la consommation des ménages.

${ }^{21}$ À l'image de Val d'Europe à Chessy qui a intégré depuis 2010 le périmètre Insee de l'agglomération.
} 


\section{Une tentative de mesure des transferts de richesses générés par les comportements de consommation des Franciliens}

Il ne saurait y avoir une approche spatiale de la consommation sans en définir une géographie. Cependant, l'étude des transferts de richesses par les pratiques spatiales soulève des difficultés méthodologiques importantes et souvent rédhibitoires. Afin de combler ce manque, notre démarche s'appuie sur le croisement original de deux enquêtes ménages. Elle repose sur l'estimation des budgets de dépenses des ménages franciliens et sur l'affectation d'un montant de dépense à chacun de leurs déplacements. Il s'agit enfin d'organiser la comptabilité des flux commerciaux à une échelle géographique en mesure de répondre aux contraintes de l'échantillonnage.

\subsection{Premières esquisses d'une géographie de la consommation}

À défaut de données sur les flux interrégionaux de dépenses de consommation, les géographes et économistes ont concentré leurs travaux sur la production d'analyses géographiques à partir de données sur l'emploi ou sur les établissements commerciaux (Berry, 1971; Beaujeu-Garnier et Delobez; 1977; Moati et al., 2004 ; Capon, 2011; Terral et Proulhac, 2014) ou encore sur les déplacements pour motif d'achat (Leo et Philippe, 2000 ; Delaporte et Courel, 2006). Ainsi, bien que structurante pour la théorie de la base, la circulation monétaire permise par les dépenses de consommation n'est que très peu documentée. Les transactions monétaires sont pourtant aujourd'hui pour une grande partie informatisées mais ces données sont tellement sensibles ${ }^{22}$ qu'il est aujourd'hui difficile, si ce n'est impossible, d'y recourir. Pour autant, cette préoccupation n'est pas récente.

Dès les années 1950, Labasse s'est intéressé aux mouvements des liquidités bancaires. Dans son ouvrage, «Les capitaux et la région » (Labasse, 1955), l'auteur observe la sensibilité de l'économie régionale aux cycles saisonniers de la production. Par le prisme de l'investissement, ses sources et ses modes de réalisation, il montre alors la contribution que peut apporter l'exploitation de ces données. Mais cette approche originale des flux monétaires n'a pas été reconduite. Il existe pourtant bien les chiffres de la comptabilité nationale qui documentent finement les dépenses de consommation par produit, toutefois ceux-ci intègrent peu la dimension géographique. En France, seule l'enquête budget des familles permet de calculer des disparités régionales de dépenses de consommation (Davezies, 2007). Ce type de données régionales reste cependant rare et de portée limitée.

En l'absence de données monétaires des flux de consommation, il existe des enquêtes locales sur les pratiques de déplacements qui peuvent approcher ces flux de façon indirecte. Ainsi, plusieurs grandes villes françaises disposent d'enquêtes de déplacements qui offrent la possibilité d'approcher l'acte de consommation au travers des rythmes urbains, des emplois du temps ou encore des trajectoires spatiales (Bailly et Heugron, 2001; André-Poyaud et al., 2006; Thévenin et al., 2007; Tabaka, 2009). Malgré tout, A. Michaud-Trévinal et G. Cliquet estiment qu'on connaît encore assez mal les parcours qui conduisent le consommateur à un point de vente tout comme le montant des dépenses qui y sont alors effectuées (Michaud-

${ }^{22}$ Pierre Veltz (2012) signale qu'en dépit d'un accroissement substantiel de la connaissance de la sphère privée, les données accessibles à la recherche publique demeurent infiniment plus réduites. 
Trevinal et Cliquet, 2002). Il existe bien quelques enquêtes estimant le montant des flux commerciaux mais celles-ci demeurent rares et confidentielles ${ }^{23}$. De plus, lorsque de telles enquêtes existent, elles ne considèrent que le stock de consommateurs domiciliés dans la zone d'étude et l'inscription de leurs pratiques d'achat dans ce même périmètre, ce qui conduit mécaniquement à éluder la question du consommateur de passage (Hermann et Beik, 1968; Papadopoulos, 1980; Jarratt, 2000; Madry, 2012). Faute de mesures empiriques, l'intensité des dépenses se mesure alors à l'aune du chiffre d'affaires et en rapport à un potentiel de consommation estimé au regard des spécificités de la population résidente.

Pour autant, il existe aujourd'hui un domaine, le tourisme, qui fait exception par la richesse de l'information disponible sur les transferts de richesse. Ce secteur s'étant imposé progressivement comme un important facteur de stimulation de l'activité régionale, il a donné lieu à la mise en place d'enquêtes spécifiques. P. Lejoux (2002) a en particulier mis en évidence l'existence d'un transfert interrégional de l'ordre de 30,3 milliards d'euros en 1999 et d'une concentration des dépenses au sein de 6 des 22 régions de France métropolitaine. Si ce type d'enquête apporte un éclairage sur l'influence de la composante touristique, elle ne renseigne en revanche rien de l'influence du consommateur de passage ${ }^{24}$. Pourtant, l'accroissement généralisé de la mobilité des populations, qu'il s'agisse des retraités, des étudiants, des actifs ou des touristes, contribue à la redistribution des revenus entre lieux de création de ces revenus et lieux de leur consommation.

\subsection{Méthodologie : le croisement de l'Enquête Budget des Familles et de l'Enquête Globale Transport}

Faute de données localisées sur les dépenses de consommation des ménages, l'analyse s'appuie sur le croisement de deux enquêtes quantitatives complémentaires. Ces bases de données renseignent d'une part les montants de dépenses de consommation, avec l'enquête Budget des Familles (BDF), et d'autre part les déplacements quotidiens des habitants d'Île-de-France, avec l'Enquête Globale Transport (EGT).

La première source utilisée est l'Enquête Budget des Familles de 2006 menée par 1'INSEE auprès des ménages ordinaires ${ }^{25}$ résidant en France. Elle fournit des estimations du revenu disponible brut ${ }^{26}$ des ménages et des dépenses annuelles de consommation pour les différents biens et services inventoriés. L'enquête constitue ainsi une source d'informations détaillées sur leurs caractéristiques, leurs ressources et leurs postes de dépenses. L'échantillon métropolitain compte 10240 ménages enquêtés, parmi lesquels 1803 résident en Île-de-France ${ }^{27}$.

\footnotetext{
${ }^{23}$ On pense ici aux estimations émanant de cabinets d'étude ou de CCI s'appuyant sur des conventions telles que les indices de disparités de la consommation qui recourent à des moyennes marginales pour estimer le montant de dépenses localisées.

${ }^{24}$ Celui qui passe mais ne séjourne pas (aucune nuitée).

${ }^{25}$ L'Insee entend par ménage ordinaire l'ensemble des personnes qui occupent un même logement au titre de leur résidence principale.

${ }^{26}$ Le revenu disponible brut tient compte de l'ensemble des sources de revenus du ménage (salaires, revenus du patrimoine, aides sociales...) diminué des impôts directs et des prélèvements sociaux.

${ }^{27}$ Les pratiques de consommation étudiées se limitent aux pratiques des Franciliens en Île-de-France. Autrement dit, les actes de consommation des Franciliens hors de leur région de résidence ne sont pas considérés. De même, les actes de consommation des non-Franciliens dans la région sont exclus du champ de l'analyse.
} 
La deuxième source mobilisée estl'Enquête Globale Transport 2010 menée par le Syndicat des Transports d'Île-de-France (STIF) et la Direction Régionale et Interdépartementale de l'Équipement et de l'Aménagement (DRIEA). Elle renseigne les caractéristiques des ménages, des individus et des déplacements quotidiens des Franciliens (modes, motifs, origine, destination...). Les données recueillies permettent de saisir assez finement les pratiques spatiales de consommation des individus. En 2010, ce sont près de 18000 ménages et plus de 42000 individus de 5 ans et plus qui ont été enquêtés en Île-de-France.

Le croisement des deux sources de données nécessite au préalable plusieurs choix méthodologiques qu'il convient maintenant de détailler. Le premier choix concerne l'échelle temporelle d'observation pour appréhender les dépenses de consommation. L'approche retenue ici est l'échelle hebdomadaire (du lundi au dimanche inclus). En effet, la littérature scientifique s'entend pour considérer cette temporalité comme fortement structurante des pratiques de consommation (Babayou et Volatier, 1998; Bondue, 2004 ; Gasnier, 2007; Chaze, 2010). Les jours de pleine semaine correspondent à des journées sous contrainte tandis que le week-end s'ouvre au règne du temps libre. Si les pratiques et les temps de consommation se partagent inégalement durant la semaine, une forme de routine s'organise néanmoins autour d'un horizon hebdomadaire. De plus, cette temporalité répond aux contraintes posées par les deux enquêtes utilisées. L'Enquête Globale Transport est effectivement construite à partir de déplacements quotidiens dont l'analyse est extensible à la semaine ${ }^{28}$. L'enquête BDF rend quant à elle possible le calcul d'une dépense hebdomadaire moyenne ${ }^{29}$.

Le deuxième choix méthodologique préalable est la définition des motifs de consommation. Dans cette analyse, la consommation est entendue comme une activité induisant un échange monétaire par lequel l'individu s'offre la jouissance d'un produit ou d'un service (Beaujeu-Garnier et Delobez, 1977; Lemarchand, 2009). Dès lors, il s'agit de définir rigoureusement dans l'EGT les motifs à destination des déplacements portés par un acte de consommation. Si l'enquête ne distingue pas spécifiquement ces déplacements, la nomenclature très fine des motifs permet cependant d'identifier ceux associés directement ou indirectement à un acte de consommation. Sur cette base, nous avons estimé que ces motifs de déplacements étaient au nombre de quatre :

- les « achats courants » qui regroupent les achats quotidiens (pain, journal...) et hebdomadaires ou bihebdomadaires (achats en grandes surfaces notamment) ${ }^{30}$;

- les « achats exceptionnels » (équipement de la maison, habillement...);

- les « loisirs et spectacles $»^{31}$;

- la « restauration » (restaurant, café...).

${ }^{28}$ Les ménages enquêtés le week-end le sont pour les deux jours qui composent le week-end. En revanche, on ne connaît leurs déplacements que pour un seul des 5 jours ouvrables de semaine. Pour cette raison, suivant les recommandations de la DRIEA, ces derniers sont pondérés par leur probabilité d'inclusion.

${ }^{29} \mathrm{Ce}$ montant moyen est donc une valeur lissée qui ne peut rendre compte des différences saisonnières (fêtes, soldes, vacances...). En revanche, on ne s'expose plus au biais de manipuler des chiffres annuels comme si les Franciliens ne prenaient jamais de vacances.

${ }^{30}$ Le guide méthodologique de l'enquête précise bien que l'activité d'achat est définie par sa périodicité. Il n'y a achat que si l'individu acquiert quelque chose. Ainsi le lèche-vitrine n'est pas considéré comme un acte d'achat.

${ }^{31}$ Pour des questions d'échantillonnage et de représentativité statistique, ces deux motifs de déplacements ont été agrégés. Aussi, se rendre à son cours de musique, aller pratiquer un sport, assister à un concert ou aller à une exposition relèvent à notre sens d'une seule et même logique. 
Il convient enfin de préciser que le poste de la « santé », pourtant renseigné dans l'enquête, a été écarté du champ d'étude en raison d'un échantillon trop réduit rendant délicate toute analyse. Une telle approche introduit de fait un biais de non-exhaustivité des dépenses de consommation, à savoir celles ne nécessitant pas de déplacement. Autrement dit, les dépenses de logement, les crédits, l'e-commerce, la vente par correspondance ou encore les diverses formes d'abonnement ne sont pas retenus dans la présente analyse.

Tableau 1 : Assignation des postes de dépenses aux motifs de déplacement

\begin{tabular}{|c|c|}
\hline Motifs EGT & Postes de dépenses BDF \\
\hline Achats courants & $\begin{array}{l}\text { Produits alimentaires et boissons non alcoolisées } \\
\text { Boissons alcoolisées et tabac }\end{array}$ \\
\hline $\begin{array}{l}\text { Achats occasionnels ou } \\
\text { exceptionnels }\end{array}$ & $\begin{array}{l}\text { Articles d'habillement et articles chaussants } \\
\text { Ameublement, équipement et entretien ménager } \\
\text { Achat de véhicules ou leurs accessoires } \\
\text { Achats d'équipements de la maison } \\
\text { Achats d'équipements de la personne }\end{array}$ \\
\hline Spectacles et Loisirs & $\begin{array}{l}\text { Services sportifs et récréatifs } \\
\text { Cinémas, théâtres, salles de concert } \\
\text { Musées, jardins zoologiques et similaires } \\
\text { Casinos, Cercles de jeux... } \\
\text { Autres dépenses de loisirs }\end{array}$ \\
\hline Restauration & $\begin{array}{l}\text { Restaurants } \\
\text { Cafés, bars et assimilés } \\
\text { Cantines }\end{array}$ \\
\hline
\end{tabular}

Source : EGT 2010, BDF 2006

Sur la base des motifs de consommation définis ci-dessus, nous avons opéré le regroupement et la ventilation par assignation manuelle des postes de dépenses correspondants de l'enquête BDF (Tableau 1). Au final, en moyenne, selon l'enquête BDF, le poids des quatre postes de dépenses retenus représente $49 \%$ de l'ensemble du budget total incombant aux ménages franciliens.

\subsection{Estimer le budget de dépenses des ménages de l'Enquête Globale Transport}

Une fois le croisement des deux enquêtes accompli, l'étape méthodologique suivante consiste à estimer de façon robuste les dépenses des ménages de l'EGT à partir de celles, connues, des ménages de l'enquête BDF.

En nous appuyant sur l'échantillon de ménages franciliens de l'enquête BDF, et d'un ensemble de variables socio-économiques communes aux deux enquêtes (tableau 2), nous avons effectué une régression linéaire par élimination descendante. Le choix du modèle linéaire s'impose en raison du caractère quantitatif et continu de la variable endogène. L'élimination descendante permet ensuite d'automatiser statistiquement la procédure d'inclusion-exclusion des variables explicatives au regard d'un niveau de significativité 
de $5 \%$. D'après l'enquête BDF, pour les quatre postes de dépenses retenus, le coefficient de corrélation ajusté de la droite de régression est de 0,56 et les valeurs du t-test sont toutes contenues dans l'intervalle de confiance usuel de $[-1,96,1,96]$.

Tableau 2 : Estimation de la valeur des paramètres du modèle

\begin{tabular}{|c|c|c|}
\hline Variable & Coefficient (t-test) & $\begin{array}{c}\text { Inflation } \\
\text { de la variance }\end{array}$ \\
\hline Constante du modèle & $3,65938(14,21)$ & 0 \\
\hline Nombre de personnes au chômage dans le ménage & $-0,15037(-4,29)$ & 1,13144 \\
\hline Chef de famille n'ayant jamais été scolarisé & $-0,23426(-5,29)$ & 1,25683 \\
\hline $\begin{array}{l}\text { Chef de famille n'ayant jamais été scolarisé au-delà } \\
\text { du primaire }\end{array}$ & $-0,23839(-4,86)$ & 1,18279 \\
\hline Revenu disponible annuel du ménage & $0,55564(21,84)$ & 1,87762 \\
\hline Nombre d'unités de consommation du ménage & $0,56728(11,77)$ & 1,64036 \\
\hline Ménage d'accédants à la propriété & $0,08207(2,28)$ & 1,39601 \\
\hline Ménage de propriétaires & $0,15555(4,2)$ & 1,58598 \\
\hline $\begin{array}{l}\text { Ménage occupant un logement à titre non } \\
\text { conventionnel }\end{array}$ & $0,3612(2,25)$ & 1,07259 \\
\hline Chef de famille agriculteur-exploitant & $-0,84034(-2,11)$ & 1,02527 \\
\hline Chef de famille employé & $-0,13023(-3,41)$ & 1,23291 \\
\hline Chef de famille ouvrier & $-0,15(-3,07)$ & 1,30845 \\
\hline Chef de famille élève ou étudiant & $-0,20023(-2,22)$ & 1,09664 \\
\hline Ménage habitant le rural francilien & $0,16118(2,03)$ & 1,04241 \\
\hline Nouveaux résidants (emménagement $<5$ ans) & $0,12405(3,92)$ & 1,16912 \\
\hline $\begin{array}{l}\text { Ménage disposant d'une surface habitable d'au } \\
\text { moins } 150 \mathrm{~m}^{2}\end{array}$ & $0,18034(3,38)$ & 1,16637 \\
\hline Chef de famille âgé de 65 ans ou plus & $-0,25688(-6,05)$ & 1,58332 \\
\hline Nombre d'observations : 1803 & \multicolumn{2}{|l|}{$\mathrm{R}^{2}=0,5612$} \\
\hline SAS Software d'après l'enquête BDF 2006 & \multicolumn{2}{|l|}{$\mathrm{R}^{2}$ ajusté $=0,5570$} \\
\hline
\end{tabular}

La régression met notamment en exergue le poids déterminant du revenu (annexe I) et du nombre d'unités de consommation (annexe II) dans l'estimation du montant de dépense d'un ménage. Les valeurs prédites qui se dégagent du modèle se distribuent bien autour de la droite de régression malgré une hausse de la variance dans les valeurs extrêmes (Figure 2). Une grande partie de celle-ci se concentre sur des ménages déclarant de faibles montants de dépenses et répondant pour partie aux propres limites de l'enquête ${ }^{32}$. Il est d'ailleurs notable qu'avec l'amélioration apportée à l'enquête de $2006^{33}$, le comportement du modèle s'améliore ${ }^{34}$.

\footnotetext{
${ }^{32}$ Pour des enquêtes aussi lourdes, attenantes à des sujets aussi sensibles que les montants de revenus et de dépenses, des biais récurrents tiennent à la sous-estimation des montants réels ou à la non déclaration de certaines dépenses.

${ }^{33}$ Échantillon plus conséquent, amélioration des méthodes de collecte et de redressement...

${ }^{34}$ Le modèle, lorsqu'il est appliqué à l'enquête BDF de 2001, justifie de propriétés similaires, avec un $\mathrm{R}^{2}$ ajusté de 0,53 et d'une distribution analogue des observations autour de la droite de régression.
} 
Figure 2 : Régression des valeurs observées par les valeurs prédites du modèle

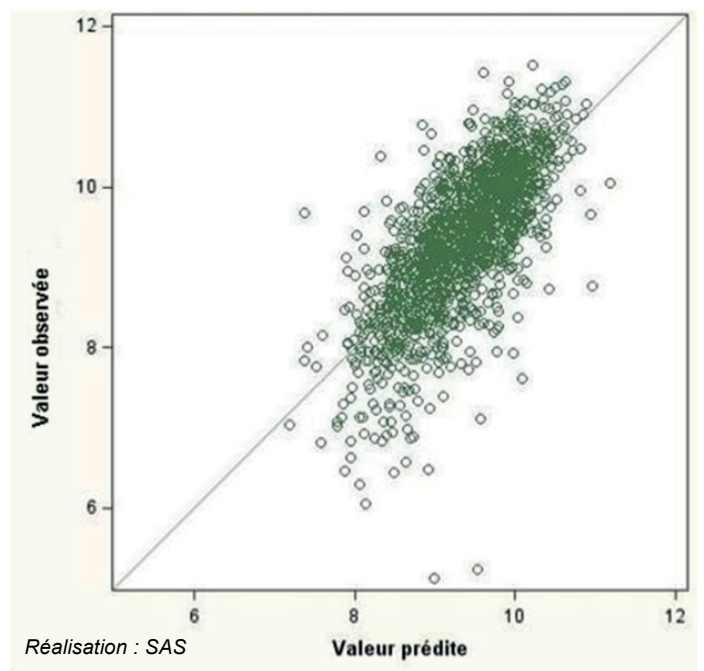

Au final, le modèle se montre robuste ${ }^{35}$, stable dans le temps et permet ainsi une estimation pertinente du budget de dépenses des ménages de l'EGT. Ce budget se désagrège ensuite selon un coefficient budgétaire propre à chacun des postes de dépenses ${ }^{36}$.

\subsection{Affecter un montant de dépense à chaque déplacement}

La méthode mise en œuvre jusqu'à présent permet d'estimer un budget, total et désagrégé par poste, de dépenses des ménages de l'EGT. Dès lors, la dernière étape méthodologique consiste à affecter un montant de dépenses à chacun des déplacements, entendus comme autant d'actes de consommation.

Pour autant, il est difficile d'estimer ce montant. Les données à notre disposition ne permettent pas de différencier par exemple l'achat d'une baguette de pain de l'achat d'un ensemble de provisions alimentaires au supermarché. Devant l'impossibilité de départager deux déplacements d'un même motif ${ }^{37}$, nous les avons considérés comme équivalent. Autrement dit, si un ménage dispose d'un budget de 100 euros hebdomadaires pour les

\footnotetext{
${ }^{35}$ Au-delà du coefficient de corrélation, des estimations de la valeur des paramètres ou de l'inflation de la variance, les principaux contrôles effectués convergent dans ce sens : distribution gaussienne des résidus, diagramme quantile-quantile, R-student...

${ }^{36}$ Le coefficient budgétaire est la part du budget d'un ménage consacrée à un poste de dépense particulier. Celui-ci va varier en fonction du niveau de vie du ménage et de ses préférences. Ainsi, fidèle à la loi d'Engel (1857), le coefficient budgétaire du poste « alimentation » tend à décroître avec le niveau de vie. À l'inverse, les loisirs et spectacles prennent une plus large place. Afin d'en tenir compte, les coefficients budgétaires sont ici établis selon le niveau de revenu du ménage et le nombre d'unités de consommation qui le composent.

${ }^{37} \mathrm{D}$ 'autres hypothèses sont envisageables, tenant compte des distances parcourues, des temps de trajet ou encore du temps de l'activité, mais aucune d'entre elles n'est véritablement convaincante. À notre sens, elles complexifient le calcul sans nous garantir de meilleurs résultats. D'autant plus, que les biais qui accompagnent ces hypothèses sont difficiles à appréhender à tout point de vue.
} 
loisirs et spectacles, que celui-ci effectue 4 déplacements à cette fin, nous considérerons qu'ils sont chacun vecteur d'une dépense de 25 euros.

Néanmoins, ce choix soulève trois biais méthodologiques notables qu'il convient de garder à l'esprit. Le premier provient du fait que la méthode ne prend pas en compte la personne qui au sein du ménage effectue l'acte de consommation. Elle neutralise par exemple l'influence spécifique du préposé aux «achats courants » qui peut exister dans un ménage, encore très souvent la femme dans les ménages en couple (Desse, 2001 ; Delaporte et Courel, 2006; Aguilera et al., 2010). Partiellement pris en compte dans l'estimation des budgets ${ }^{38}$, un deuxième biais tient au postulat implicite que les ménages accèdent à un même type d'offre et rencontrent un indice des prix constants partout dans la région Île-de-France. Il est raisonnable d'envisager qu'un certain nombre de produits ou services marchands, bien qu'identiques, puissent être plus coûteux à Paris qu'en périphérie et inversement ${ }^{39}$. Enfin, un troisième provient du fait que la méthode surestime très certainement les dépenses locales. En s'affranchissant complètement de la distance, l'hypothèse rejette la rationalité économique qui pousse le consommateur à parcourir une plus grande distance afin de regrouper ses achats et en augmenter ses volumes. Au final, l'hypothèse retenue est une hypothèse conservatrice qui minimise à notre sens le risque de surestimation des transferts de richesses interdépartementales.

Cette approche donne non seulement une mesure approximative des dépenses de consommation mais dégage également un cadre inédit d'observation des transferts de richesses en Île-de-France. Là où les travaux de l'IAURIF renseignent la fréquentation des départements pour le motif achat (Delaporte et Courel, 2006), cette comptabilité propose un éclairage sur des actes de consommation plus variés et leur valorisation monétaire.

Tableau 3 : Valorisation monétaire moyenne d'un déplacement selon le revenu du ménage

\begin{tabular}{|cc|}
\hline Classe de revenus & Montant par déplacement \\
\hline$<€ 9600$ & $39 €$ \\
$€ 14400<19200$ & $66 €$ \\
$€ 19200<24000$ & $75 €$ \\
$€ 24000<28800$ & $89 €$ \\
$€ 28800<36000$ & $119 €$ \\
$€ 36000<42000$ & $149 €$ \\
$€ 42000<54000$ & $181 €$ \\
$€ 54000<66000$ & $207 €$ \\
$>€ 66000$ & $224 €$ \\
\hline
\end{tabular}

Source : BDF 2006, EGT 2010

Le tableau 3 montre à titre d'exemple qu'il faut environ dix déplacements de consommation d'un ménage du $1^{\mathrm{er}}$ décile pour espérer capter un montant de dépenses équivalent

\footnotetext{
${ }^{38}$ L'estimation du budget tient compte du tissu urbain de résidence et distingue notamment les citadins qui vivent en agglomération des périurbains.

${ }^{39}$ Le marché de seconde main est d'un rare dynamisme à Paris et présente un grand nombre de biens et de services peu coûteux au regard des standards du périurbain francilien.
} 
à celui d'un seul déplacement d'un ménage du dernier décile. Cette comptabilité indicative est de surcroît confortée dans sa vraisemblance par la concordance de la distribution des dépenses ainsi obtenue avec celle des emplois domestiques ${ }^{40}$ (Ruault, 2012).

\subsection{Sélectionner une échelle géographique utilisable et pertinente}

Afin de préserver la significativité des résultats, l'analyse ne peut s'opérer qu'au sein de grands agrégats géographiques. Parmi les mailles administratives, seul le périmètre des huit départements franciliens offre des garanties suffisantes. On y compte entre 200 et 300 ménages enquêtés le week-end, et 1000 à 1700 en semaine. Il n'est en revanche pas possible d'affiner cette analyse à l'échelle des bassins de vie ou des zones d'emploi. Une alternative intéressante réside néanmoins dans une approche morphologique, partageant Paris, la banlieue et le périurbain (Ruault, 2012), mais telle n'a pas été notre volonté ici. D'une part, l'existence et la permanence d'espaces fonctionnels que l'on puisse délimiter, à l'instar des bassins de vie, sont discutables (Vanier, 2008; Massot et al., 2009). De tels espaces reposent sur des arbitrages statistiques instables, de par leur nature normative, et parce que leurs périmètres évoluent dans le temps. D'autre part, même si les départements imposent des contours largement fictifs, ces contours sont immuables et correspondent aux frontières d'espaces au sein desquels se construit l'action publique. De fait, ce sont aujourd'hui les commissions départementales d'aménagement commercial ${ }^{41}$ (CDAC) qui statuent sur les demandes d'ouverture des grandes surfaces et le périmètre des Schémas de Développement Commercial (SDC) est bien celui du département. De surcroît, un nombre grandissant de départements endosse aujourd'hui un rôle de médiation auprès des acteurs locaux et cherche à organiser l'action publique autour de projets de territoire. Les représentations du développement et des horizons souhaitables pour l'économie francilienne émanent pour partie de ces institutions. Or c'est la connaissance qui conditionne ces représentations et l'action publique qui en découle. En empruntant des périmètres qui sont inscrits durablement dans le paysage statistique et institutionnel, qui font sens pour les décideurs locaux, nous cherchons ici à attirer l'attention sur des transferts de richesses jusqu'alors non étudiés.

\section{Résultats : les premiers jalons d'une géographie des dépenses de consommation en Île-de-France}

Selon que les systèmes de mesure interviennent en amont ou en aval du flux circulatoire, un même territoire peut être entendu comme plus ou moins riche et performant. Aux mesures classiques de PIB et de revenus, on peut également s'intéresser à l'aptitude des territoires à valoriser localement la demande endogène émanant des consommateurs présents sur le territoire. Enfin, les résultats cherchent à mettre en évidence la consommation francilienne comme moteur de développement local et comme vecteur de liens d'interdépendances économiques.

\footnotetext{
${ }^{40}$ Les emplois domestiques sont généralement utilisés comme proxy des dépenses de consommation qui les soutiennent.

${ }^{41}$ Plus précisément, les CDAC réunissent des élus et des personnes qualifiées afin de statuer sur les demandes d'autorisation d'urbanisme des équipements commerciaux de plus $1000 \mathrm{~m}^{2}$.
} 


\subsection{Une autre lecture de la distribution spatiale des richesses}

Les enjeux du développement territorial peuvent embrasser des réalités très différentes selon les acteurs territoriaux, leurs priorités, et le contexte spatio-économique. Le rayonnement et la compétitivité d'un territoire à l'échelle nationale ou internationale peuvent ainsi constituer des enjeux territoriaux forts. La capacité endogène d'un territoire à construire des marchés et à satisfaire les attentes localement peut en constituer d'autres. Enfin, les conditions de vie des habitants, l'importance des capitaux dont ils disposent, sont également déterminants. Pourtant ces enjeux ne se combinent pas nécessairement. Or la mesure de la richesse et de la performance économique n'a pas le même sens selon l'approche privilégiée. S'agit-il de mesurer la création de biens et de services marchands (richesses)? De mesurer la capacité d'un territoire à en créer toujours plus? Ou bien est-il plutôt question d'adéquation entre offre locale et demande endogène, et ainsi d'une mesure de la valorisation effective qui est faite des capitaux qui transitent en un territoire? Ou encore la richesse se mesure-t-elle à la profondeur des comptes en banque et du patrimoine de ses habitants, de l'évolution des richesses détenues au fil du temps et des peuplements?

La richesse des territoires est traditionnellement approchée par le produit intérieur brut (PIB), en tant qu'indicateur de la création locale de richesses, ou par les revenus $(\mathrm{RNI})^{42}$, qui dénotent alors du niveau de vie des habitants. En revanche, l'aptitude des territoires à mettre en circulation des richesses sur leur marché local par la dépense des ménages est rarement et partiellement étudiée au travers des seules recettes touristiques $(\mathrm{DCT})^{43}$. Au regard des dernières données disponibles, la figure III propose de remettre en perspective ces trois mesures, au travers d'une lecture comparative, introduisant un quatrième indicateur: les recettes commerciales provenant de la consommation des ménages franciliens (DCR $)^{44}$. Il s'agit tout simplement de la somme des dépenses franciliennes enregistrées à destination des départements d'Île-de-France tel qu'il a été possible de les estimer. De la sorte, l'assiette des recettes commerciales étudiées tend à avoisiner la réalité du marché, c'est-à-dire l'ensemble des dépenses des personnes effectivement présentes en Île-de-France ${ }^{45}$.

42 À défaut de données départementales sur le revenu disponible brut, nous utiliserons le revenu net imposable (RNI) ici entendu comme l'ensemble des revenus perçus par les ménages, avant abattement fiscal et après déduction des pensions, déficits et pertes de capital, auquel on retranche l'impôt sur le revenu.

${ }^{43}$ Les recettes touristiques sont abordées par les volumes de dépenses de consommation touristique (DCT) enregistrés au lieu de séjour.

${ }^{44}$ Les dépenses de consommation régionale (DCR) par habitant ne couvrent pas l'ensemble des postes de dépense des ménages et se limitent aux quatre postes retenus par la méthode mise en place : achats courants, achats exceptionnels, spectacles -loisirs et restauration.

${ }^{45}$ Seuls les excursionnistes non franciliens échappent au radar. Vu qu'ils ne séjournent pas, ils ne sont pas comptabilisés parmi les touristes, et vu qu'ils ne résident pas en Ile-de-France, ils n'ont pas pu être intégrés aux estimations. 
Figure 3 : Mesure comparative de la performance économique en Île-de-France

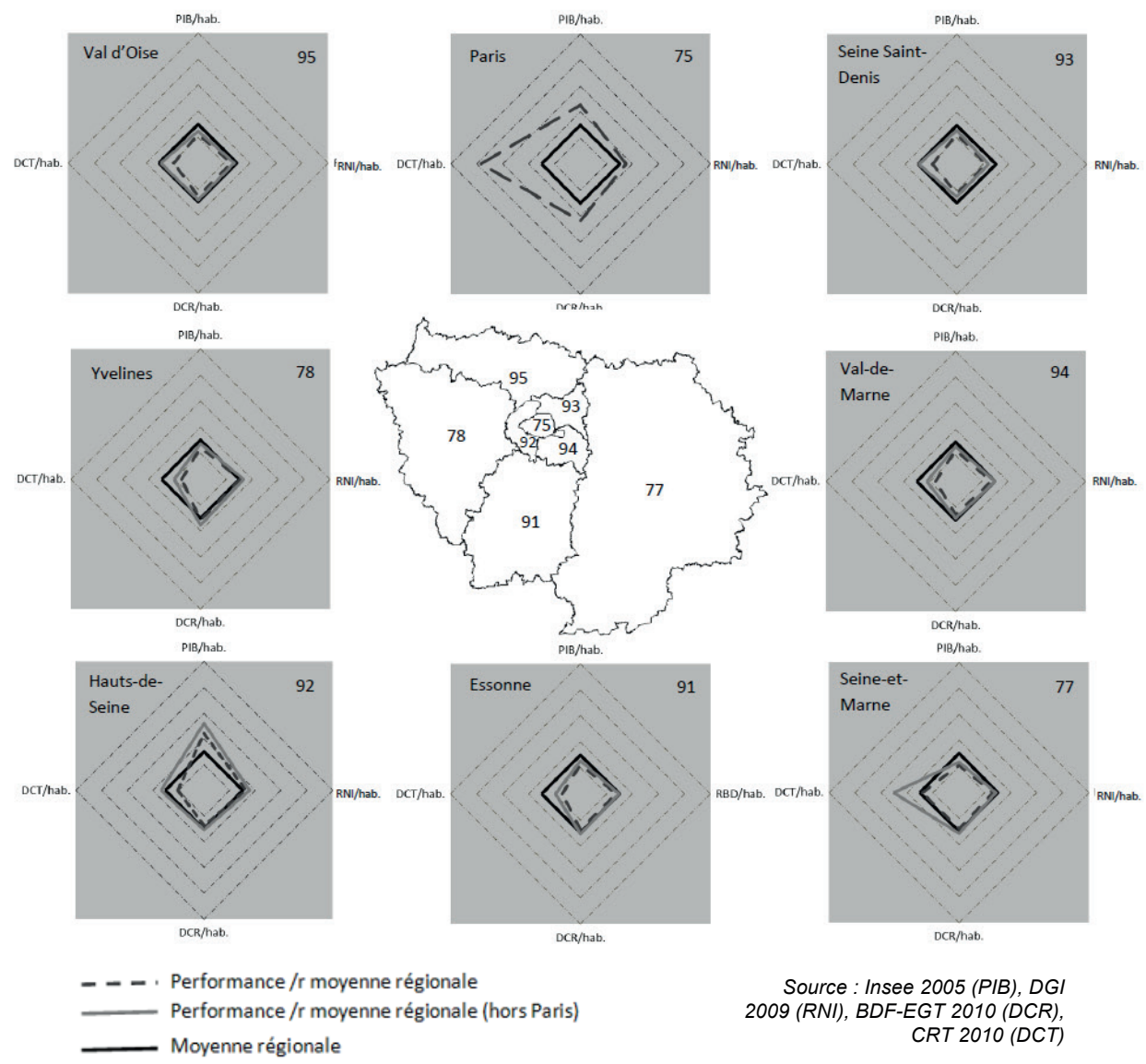

En 2010, les quatre postes de dépense étudiés pour les DCR alimentent des activités comptant pour 828000 emplois salariés privés en Île-de-France, soit $25 \%$ de l'emploi ${ }^{46}$. S'intéresser aux transferts de richesses induits par les dépenses de consommation revient donc ici à s'intéresser à ce qui engendre près d'un emploi salarié privé sur quatre en Île-de-France. Ces secteurs d'activité justifient par ailleurs d'une importante croissance de l'emploi depuis 2001 (+6\% entre 2001 et 2010), supérieure à celle de l'emploi global (+2\%).

Toutefois, le cas parisien étant sans commune mesure et afin d'améliorer la lecture comparative, les quatre indicateurs sont observés non seulement au regard de la moyenne en Île-de-France mais également à l'aune d'une moyenne régionale hors Paris. Dès lors, si les mesures de la richesse sont différentes, la hiérarchie régionale change-t-elle?

\footnotetext{
${ }^{46}$ Chiffre obtenu à partir des données Unedic sur la base de la nomenclature NAF utilisée pour l'agrégation des dépenses BDF.
} 


\subsubsection{Paris en chef de file}

Paris est un cas très spécifique. C'est le principal moteur de l'économie régionale. Paris est le grand foyer de population d' ̂lle-de-France avec plus de 2,2 millions d'habitants (soit $19 \%$ de la population francilienne) mais représente surtout un puissant moteur productif avec plus de 160 milliards d'euros de PIB estimés pour 2005 (soit $34 \%$ de la production d'Île-de-France). En outre, la population y est en moyenne $26 \%$ plus riche qu'ailleurs dans la région et agglomère près de $25 \%$ du volume de revenu net imposable francilien. Un poids qui, du fait d'importants mécanismes redistributifs (Davezies, 2007), se révèle de moindre importance que sa contribution productive mais qui reste encore très favorable. La capitale est de surcroît le pôle touristique majeur de la région Île-de-France avec la visite de plus de 28 millions de touristes en 2012 et la concentration de près des deux tiers des dépenses touristiques franciliennes. C'est également le principal centre de consommation des Franciliens, puisque $32 \%$ des dépenses régionales y sont enregistrées, mais il est remarquable que sur ce plan les inégalités interdépartementales sont substantiellement plus réduites. Au final, la Paris se présente comme une immense concentration de richesses à tous les pointages.

\subsubsection{Les Hauts-de-Seine : une difficulté à capter les consommateurs}

Le cas des Hauts-de-Seine est intéressant car caractérisé par un défaut d'attractivité notable auprès des consommateurs franciliens. Ce département de près d'1,6 millions d'habitants est le deuxième plus gros contributeur au PIB régional derrière Paris avec $23 \%$ de la production francilienne. Bien que détenteur d'une solide seconde place dans la hiérarchie régionale, les Hauts-de-Seine accusent une contre-performance notable dans la captation des dépenses de consommation. Il s'agit du département le moins performant en la matière. En moyenne, un tiers des dépenses des habitants n'est pas valorisé sur le marché local. D'après nos calculs, lorsque le territoire capte 100 euros de l'extérieur, les alto-séquanais en dépensent 240 euros en dehors du département. Sous un autre angle, ce manque d'attractivité relative à l'Île-de-France ne coïncide pas avec un déficit d'attractivité touristique. Après Paris, et avant le département de Seine-et-Marne, ce sont les Hauts-de-Seine qui tirent avantage du tourisme.

\subsubsection{La relativité de la mesure dans le cas francilien}

La contre-performance des Hauts-de-Seine est plus qu'un simple élément de diagnostic. Elle témoigne de la relativité de toute mesure et des hiérarchies simples. En économie régionale, il est possible de se donner différentes représentations de la richesse, ou du développement économique, et finalement aboutir à des conclusions différentes. À l'aune des dépenses de consommation franciliennes, un département réputé riche et performant comme celui des Hauts-de-Seine peut ainsi se montrer moins à son avantage.

La mobilisation simultanée des quatre indicateurs évite notamment d'aboutir à des résultats trop segmentés et clivant entre les territoires. En effet, les départements franciliens justifient de situations contrastées, cumulant des indices favorables et moins favorables selon les mesures. Par exemple, la Seine-et-Marne est la lanterne rouge du PIB par habitant. Et si le département se repositionne en termes de revenu, ce n'est véritablement qu'à l'aune de ses

\footnotetext{
${ }^{47}$ Et même 44 \% plus riches si l'on considère la moyenne régionale hors Paris.
} 
performances en matière de consommation régionale et touristique qu'il est possible d'identifier une force. Il en va de même pour le département de l'Essonne, au profil similaire.

Avec cette approche comparative, les situations de performances ou de défaillances cumulatives n'en prennent que plus d'ampleur. Le cas de Paris est particulièrement marquant à cet égard mais celui de la Seine Saint-Denis l'est tout autant. Le département est effectivement en retard sur tous les fronts. Pour aucun des quatre indicateurs, il ne se place au-dessus de la moyenne régionale. Ce n'est pourtant pas le département le plus modestement doté en centrescommerciaux, bien au contraire (Delaporte, 2003). En revanche, il affiche le plus grand taux de vacance des locaux commerciaux d'Île-de-France (Crocis, 2012), en particulier au sein des nombreux quartiers sensibles qui le composent. La Seine Saint-Denis cumule ainsi des difficultés sans jamais pouvoir se repositionner par rapport aux autres départements franciliens.

Autant d'enrichissements, de contrastes territoriaux, qui finalement nourrissent le besoin d'aller plus loin. En particulier, il n'est pas anodin de s'interroger sur l'origine des consommateurs franciliens qui font la richesse de départements comme la Seine-et-Marne ou les Yvelines. S'agit-il d'une meilleure aptitude de ces territoires à retenir et valoriser les dépenses de leurs habitants? S'agit-il plutôt de l'attraction de consommateurs franciliens de passage?

\subsection{Propension à consommer localement et attractivité commerciale}

Une mesure d'usage pour rendre compte de l'aptitude d'un territoire à faire dépenser ses habitants est la propension à consommer localement. Il s'agit d'apprécier dans quelle mesure les habitants mobilisent leurs richesses pour les mettre en circulation dans leur département de résidence.

\subsubsection{Rétention et valorisation des dépenses}

La carte 1 présente une estimation de la rétention locale ${ }^{48}$ des dépenses de consommation des habitants des huit départements franciliens.

Le négatif de cette mesure est l'évasion des dépenses de consommation. Plus la rétention est proche de 100, plus la propension des habitants à consommer localement est forte. On constate que ce sont les Parisiens qui dépensent le plus localement. En moyenne, sur 100 euros dépensés, 93 euros le sont à Paris. Les Parisiens sont les consommateurs franciliens qui effectuent le plus grand nombre de déplacements. Leur mobilité quotidienne est cependant très fortement ancrée à Paris intramuros. De fait, près de $70 \%$ des actifs parisiens travaillent à Paris ${ }^{49}$ (Aguiléra et al., 2009). On ne peut ignorer également que l'offre commerciale y est plus dense et diversifiée que dans le reste de l'Île-de-France, assurant de la sorte les conditions d'une meilleure adéquation entre l'offre et la demande.

Ce sont ensuite les départements de grande couronne qui témoignent de la meilleure rétention des dépenses de consommation de leurs habitants. Si on ne peut ignorer l'effet taille qui les préserve de grands flux d'évasion commerciale, il fait peu de doute que cette situation privilégiée tient également à leur éloignement géographique du centre. Autrement dit, cette distance les préserve de la concurrence de l'offre parisienne. La

${ }^{48}$ La rétention est entendue dans ce travail comme la capacité des territoires à retenir les dépenses de consommation des résidents du département. Son corollaire est l'évasion commerciale.

${ }^{49}$ En 1999, la part des actifs travaillant dans leur département de résidence est de l'ordre de $70 \%$ des actifs pour Paris, alors qu'en moyenne cela ne concerne que $58 \%$ des actifs de grande couronne et $51 \%$ de ceux de petite couronne. 
Seine-et-Marne tutoie même les $90 \%$ de rétention commerciale et, sans rivaliser avec Paris, creuse un écart avec les autres départements franciliens. Une part très importante des Seine-et-marnais réside pourtant sur la frange ouest du département et se retrouve ainsi directement exposée à l'influence de Paris.

Carte 1 : Rétention des dépenses de consommation selon le département de résidence

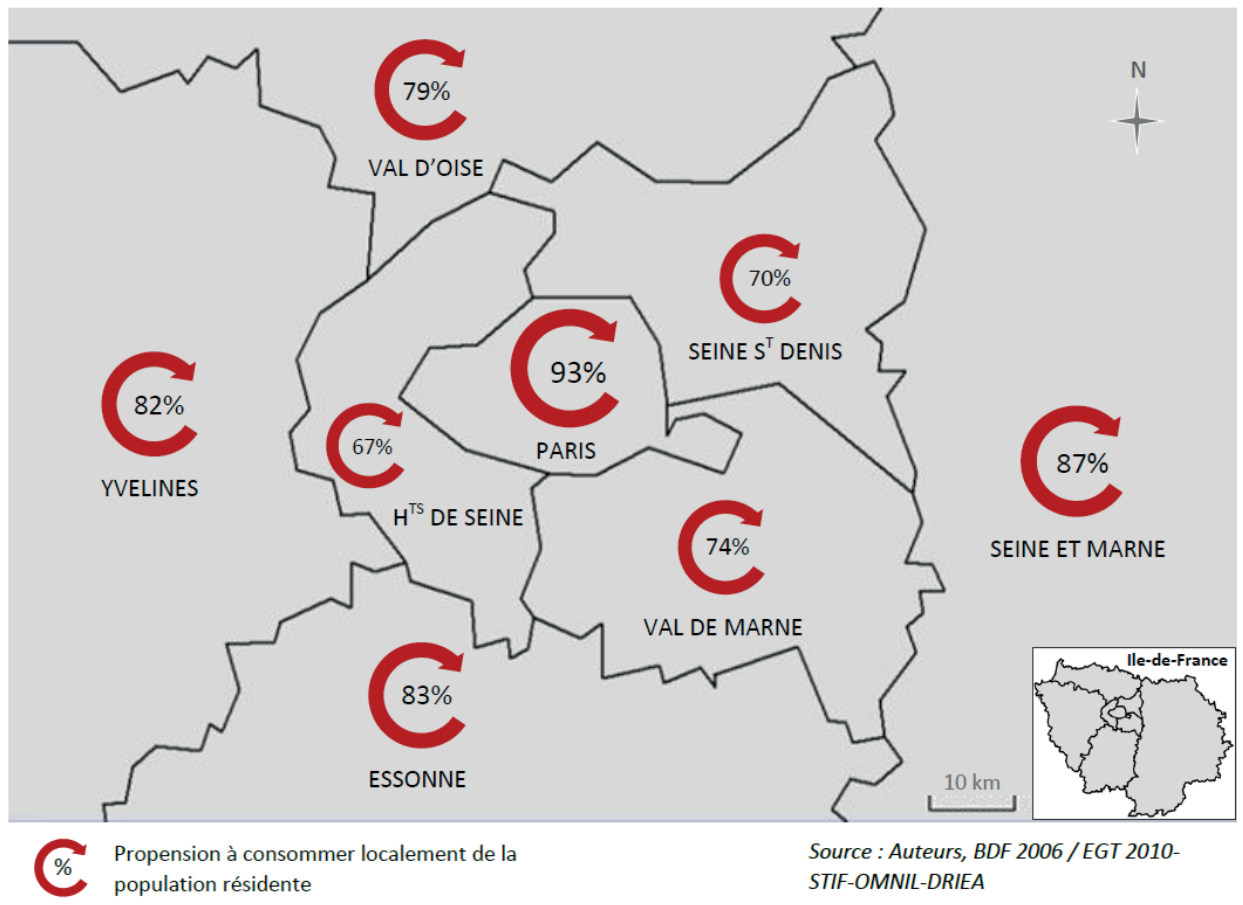

À l'inverse, et de façon très marquée, les Altoséquanais sont moins ancrés à leur marché départemental et ne consacrent que $67 \%$ de leur budget à des dépenses locales. Cela se vérifie pour chacun des quatre postes étudiés. Un retrait qui cache pourtant une nette progression depuis 2001 où la rétention ne dépassait pas les 58 euros. L'offre départementale s'asphyxie probablement par la concurrence directe avec Paris et les centres commerciaux des Yvelines. Pris en étau entre l'offre parisienne particulièrement étoffée et une ceinture de centres commerciaux régionaux de grande couronne, c'est l'ensemble des départements de petite couronne qui souffrent d'une plus grande vulnérabilité à l'évasion commerciale. En dehors du cas des Hautsde-Seine, on note par exemple que sur 100 euros dépensés par les Val-de-marnais dans les loisirs et spectacles, plus du tiers s'évadent du département. Ou bien encore que seulement 41 euros sur 100 dépensés dans la restauration ${ }^{50}$ par les habitants de Seine-Saint-Denis profitent au marché départemental.

\footnotetext{
${ }^{50} \mathrm{Il}$ est à noter que le motif « restauration » contient les repas au lieu de travail et se trouve donc fortement affecté par la localisation des grands pôles d'emploi en Ile-de-France.
} 
Au final, et de manière peu évidente, la propension à consommer localement se révèle être l'une des grandes variables explicatives de la performance commerciale de Paris. La consommation des Parisiens n'entraîne en effet que de faibles évasions de richesses et, par conséquent, une forte valorisation des revenus. La propension à consommer localement explique également le bon positionnement des départements de grande couronne dans la hiérarchie régionale qui, au contraire de la petite couronne, justifient de très fortes rétentions des dépenses de leurs habitants.

Les recettes commerciales départementales ne sauraient cependant s'expliquer au seul regard de la propension à consommer localement. Une autre dimension importante réside dans l'attraction de consommateurs de passage, ultime figure d'une approche présentielle de l'économie.

\subsubsection{L'attractivité commerciale ou le solde net des transferts interdépartementaux}

L'attractivité des départements les uns par rapport aux autres peut se mesurer à leur capacité à capter des dépenses du consommateur de passage plus vite qu'ils n'en perdent par l'évasion commerciale. Le consommateur visé ne résidant pas sur le territoire, ses dépenses constituent une injection d'euros nouveaux sur le marché local et sont des richesses basiques au même titre que la base résidentielle ou sociale. La carte 2 traduit donc le solde net des échanges commerciaux entre les pertes engendrées par l'évasion commerciale et les bénéfices provenant de l'attraction de consommateurs de passage. L'écrasante attractivité de Paris rend négatif le bilan des autres départements franciliens.

Carte 2 : Solde net des échanges commerciaux par tranche de 100 euros d'évasion commerciale

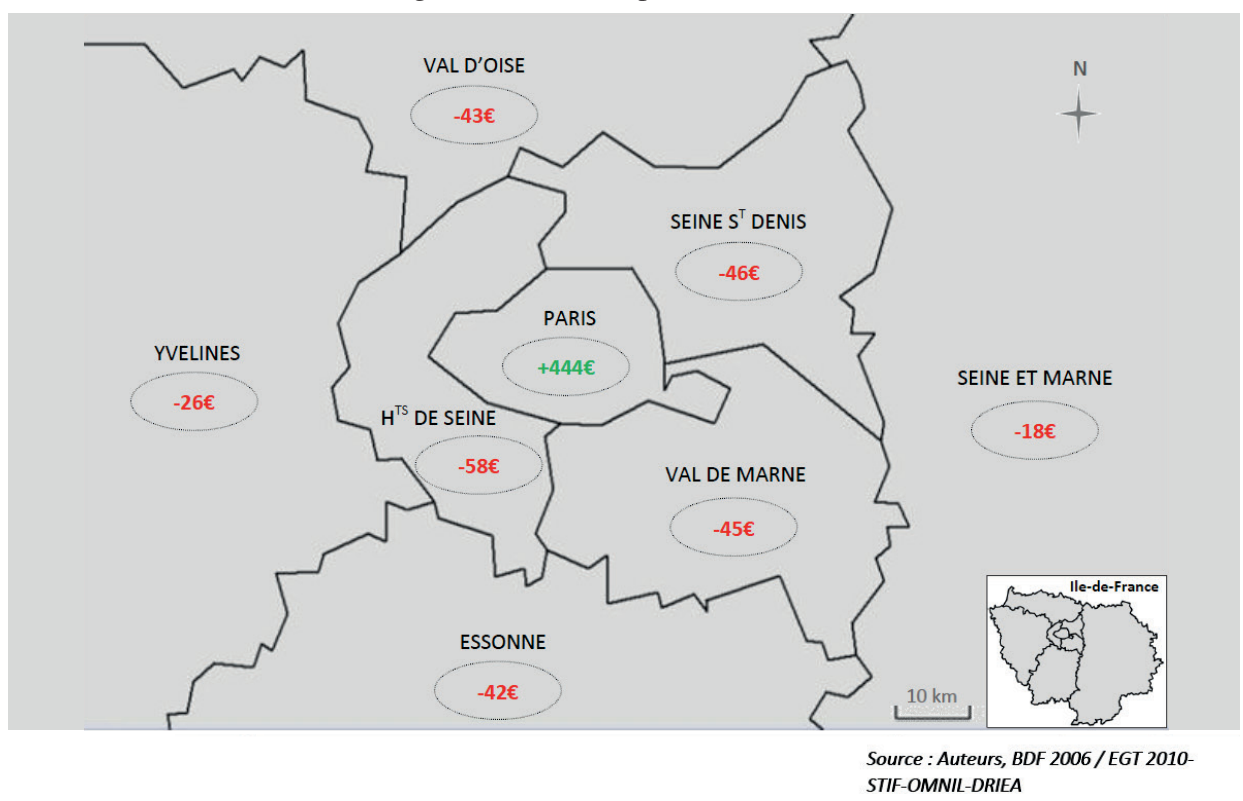

Ainsi lorsque 100 euros sont dépensés par des Parisiens en dehors de leur département de résidence, Paris enregistre un solde favorable de 444 euros grâce aux dépenses entrantes. Cette attractivité se vérifie pour tous les postes budgétaires mais particulière- 
ment pour les achats exceptionnels $(+567 €)$, les spectacles $(+330 €)$ et la restauration $(+524 €)$. Il est facile ici d'y voir l'avantage substantiel procuré par le dimensionnement et la diversité de l'offre commerciale proposée à Paris. Cette mesure illustre le mécanisme de re-captation de richesses à l'œuvre dans certaines agglomérations (Bowles, 2007). De fait, alors que les transferts de masses salariales tendent à profiter à la périphérie au détriment des villes-centres, ces dernières, par la qualité et la diversité de leur offre commerciale, attirent en retour les consommateurs de la périphérie (Davezies, 2011).

Le cas de la Seine-et-Marne est lui aussi particulièrement intéressant car c'est le département francilien qui se défend le mieux derrière Paris. Elle présente en particulier un solde positif pour les achats courants $(+37 €)$ et demeure honorablement placée pour les achats exceptionnels $(-6 €)$. Il faut dire que le département a connu une importante densification commerciale depuis les années 1990 sur sa frange ouest puisque quatre des cinq centres commerciaux régionaux y ont été créés : Val d'Europe à Serris, Carré Sénart à Lieusaint, le Carrefour de Villiers-en-Bières et Chelles 2 (Delaporte, 2003). Ce front commercial est particulièrement bien situé et attractif auprès des consommateurs de petite couronne. Il est par ailleurs renforcé par l'attractivité d'Eurodisney qui s'est implanté à Chessy en 1992 et qui attire des millions de visiteurs chaque année.

Parmi les quatre départements en deçà de la moyenne régionale, seul le département des Hauts-de-Seine décroche véritablement. En moyenne, il ne compense pas la moitié des pertes engagées par l'évasion commerciale, voire pas mieux qu'un tiers de l'évasion liée aux achats exceptionnels et aux loisirs et spectacles (-68 €). Il est en outre remarquable qu'un département comme l'Essonne, présentant une forte propension à consommer localement $(83 \%)$, supérieure même à celle des Yvelines, enregistre finalement un solde nettement défavorable. Par conséquent, si une forte propension à consommer localement peut s'accompagner d'une vraie attractivité commerciale, rien n'est mécanique. Là encore l'usage de plusieurs indicateurs ajoute du contraste à l'analyse et enrichit la compréhension du développement économique. Certains territoires comme Paris ou la Seine-et-Marne sont très attractifs, satisfaisant tout à la fois leurs habitants et une demande plus régionale. L'inverse peut se vérifier également avec les Hauts-de-Seine. Mais il existe également des profils moins tranchés où les habitants consomment beaucoup localement sans pour autant que le territoire suscite de la consommation de passage. Existerait-il des freins à la mobilité qui rendraient les habitants captifs d'une offre peu développée? Y-a-t-il des seuils d'attractivité de l'offre en dessous desquels il n'est pas possible d'attirer un consommateur éloigné ? Ce qui est certain, c'est que l'économie d'un territoire revêt de multiples dimensions qu'il est difficile de résumer en une seule mesure.

Cependant, si les mesures comptables restent relatives et mouvantes, il existe quelque chose d'assez immuable au fonctionnement économique d'un territoire, ce sont les interdépendances économiques sur lesquelles il s'appuie. En effet, le bilan comptable d'une analyse économique peut changer avec le temps et selon les modalités de mise en œuvre. L'intensité d'une relation interterritoriale également. En revanche, le lien en lui-même, l'interdépendance qui unit un à un des territoires, des filières économiques, des lieux de vie, est vraisemblablement plus constant d'où l'intérêt d'en saisir la portée. 


\subsection{Consommation de passage et liens interdépartementaux}

Les politiques publiques en faveur du développement territorial s'attachent peu au potentiel économique de la consommation de passage. A contrario, des efforts considérables sont menés, aussi bien au niveau régional qu'à l'échelle communale, afin de développer et promouvoir le tourisme. La figure 4 montre que la contribution du tourisme (CRT Paris Îlede-France, 2011) à l'économie des départements franciliens est cependant loin de dépasser la contribution du consommateur de passage, et a fortiori du consommateur local.

Figure 4 : Distribution géographique des dépenses de consommation selon le type de consommateurs

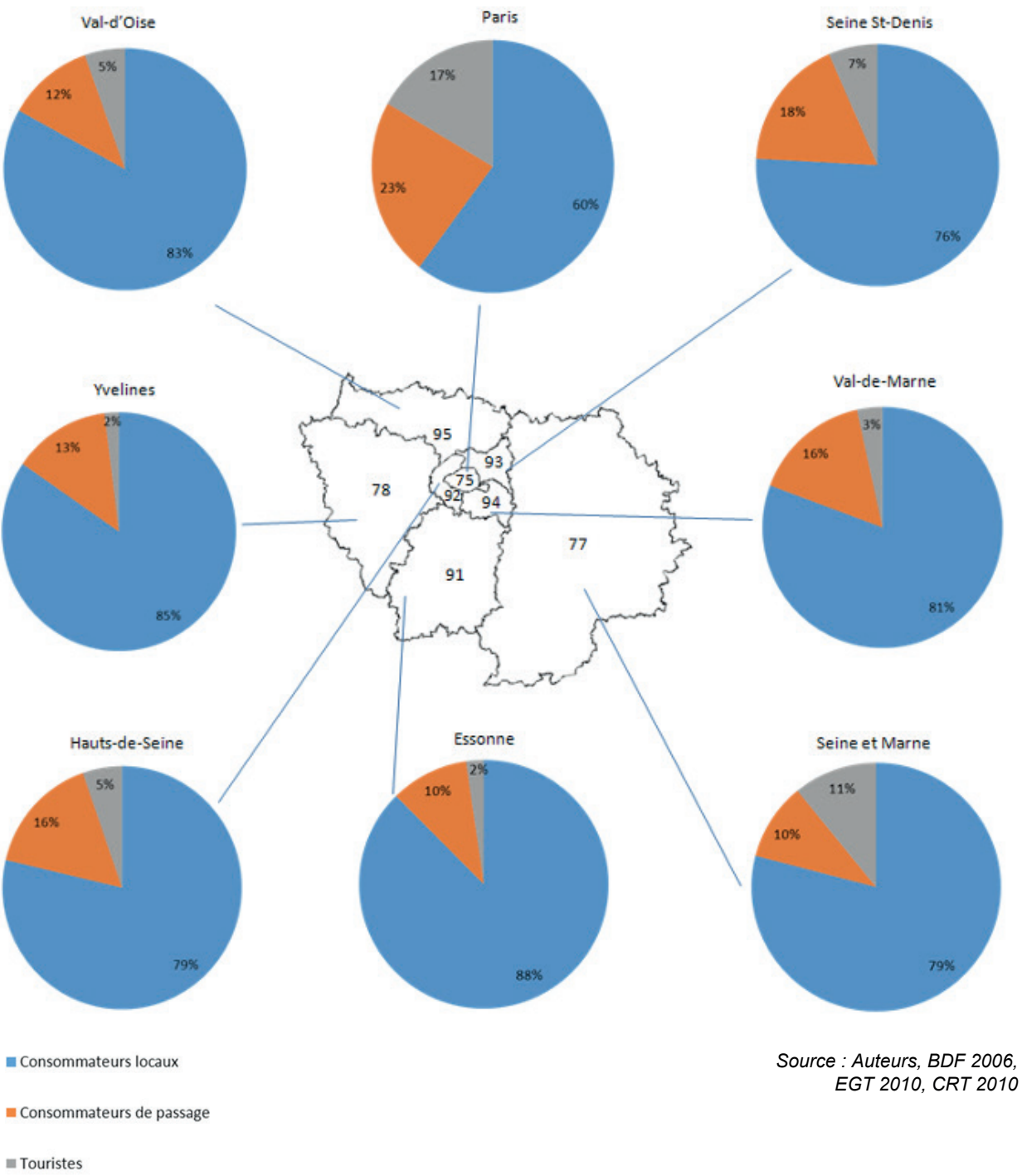


L'apport du consommateur de passage représente de fait plus de deux fois l'apport touristique à l'échelle régionale. Pour un département comme l'Essonne, c'est cinq fois l'apport touristique et pour Paris, troisième ville touristique mondiale, 1,6 fois l'apport touristique. Autrement dit, cette dimension relativement invisible de la distribution des dépenses de consommation n'en demeure pas moins puissante.

Un seul département francilien est aujourd'hui en mesure de faire mentir cette règle : la Seine-et-Marne. Les retombées économiques de Disney y sont pour beaucoup et probablement demeurent-elles encore sous-estimées ${ }^{51}$. On notera également l'accroissement du poids de la consommation de passage à mesure qu'on se rapproche du centre. Les départements de petite couronne sont ainsi les seuls départements, avec Paris, où la consommation de passage représente plus de $15 \%$ de l'économie présentielle. En somme, après le consommateur local, et avant le touriste, le consommateur de passage se présente comme un important moteur de développement économique au niveau départemental.

Or la consommation de passage n'existe qu'à l'aune des mobilités ordinaires du francilien ou de l'excursionniste. La carte 3 présente ainsi les principaux flux commerciaux qui lient les départements entre eux.

Carte 3 : Consommation des Franciliens et principaux transferts de richesses interdépartementaux

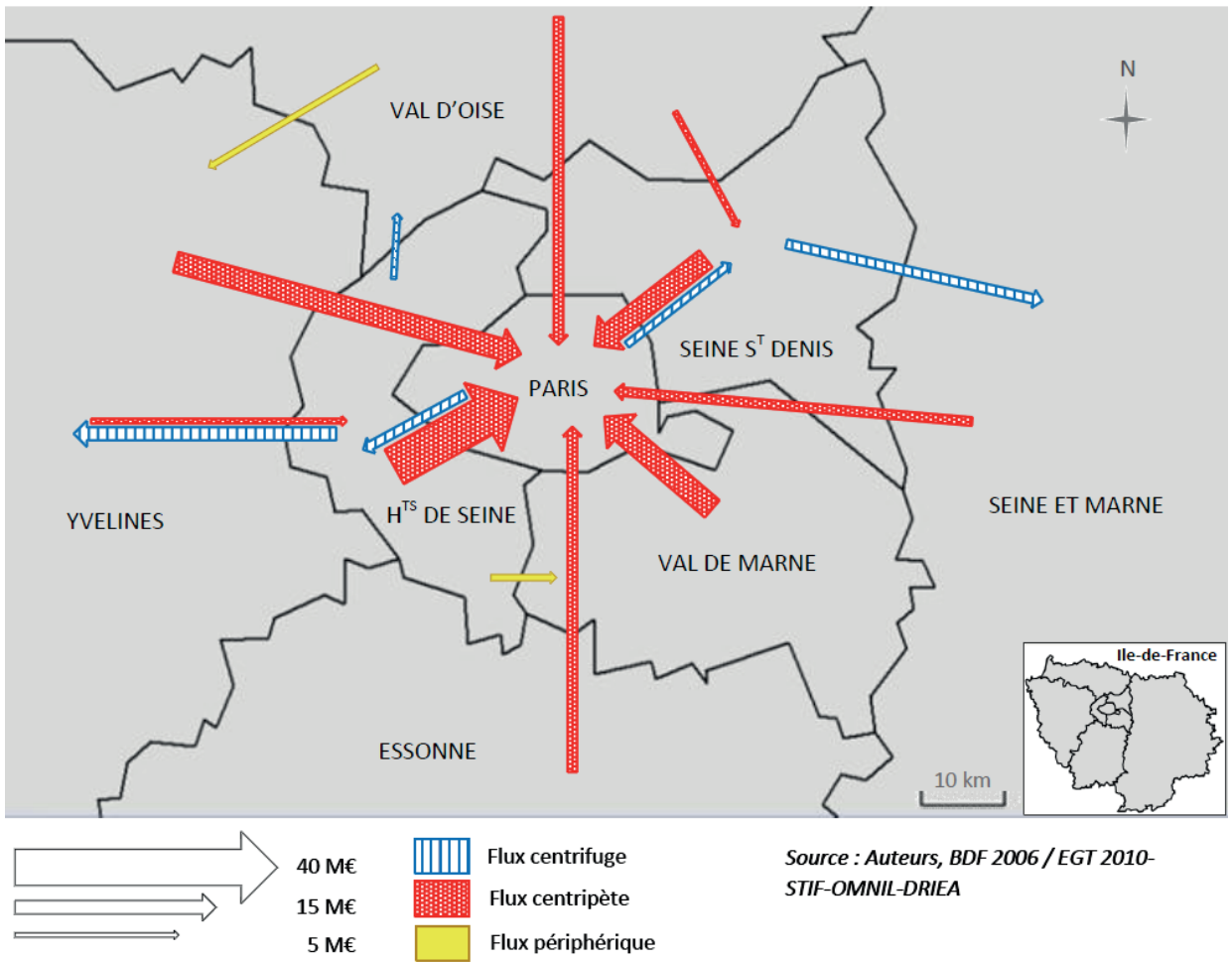

${ }^{51}$ Les chiffres du CRT Paris Île-de-France sont établis au lieu de séjour du touriste. De ce fait, les dépenses d'un touriste en séjour à Paris demeureront affectées à Paris même s'il se rend pour la journée à Disney. 
À ce titre, on observe que l'intensité des échanges varie considérablement et se révèle le plus souvent dissymétrique. Ainsi $17 \%$ de l'ensemble des transferts de richesses régionaux proviennent de la relation Paris - Hauts de Seine dont les $4 / 5^{\mathrm{e}}$ à destination du premier. Paris est de fait le département bénéficiaire des principaux flux de richesses interdépartementaux et alimente par rebond une dynamique centripète dominante. À l'inverse, les flux centrifuges témoignent de l'existence de ruptures et de liens plus circonstanciels entre départements. Les couples Yvelines - Hauts-de-Seine d'une part, et Seine et Marne - Seine-Saint-Denis d'autre part, illustrent le pouvoir d'attraction qu'exerce l'offre commerciale grandissante de seconde couronne sur les populations de première couronne. Les flux périphériques semblent quant à eux plus secondaires.

Si ces flux interdépartementaux ont des orientations différentes, ils relèvent également de motivations distinctes. Lorsqu'on écarte les dépenses exceptionnelles, assez composites, la restauration et les spectacles-loisirs se présentent ainsi comme les deux grandes motivations qui poussent les habitants de la périphérie à se rendre à Paris. À l'inverse, on note que les Parisiens se rendent principalement en périphérie pour les courses courantes et les achats exceptionnels. Autant de résultats qui invitent à élargir les champs d'investigation de l'économie régionale et soulèvent des attentes d'approfondissement des ressorts de l'économie présentielle.

\section{Conclusion}

À l'heure où se discute la mise en place de la future métropole du Grand Paris, où les départements franciliens s'apprêtent à partager un fonds de solidarité de 60 millions d'euros pour 2014, il est important de rappeler la réalité des mécanismes de marché qui prennent place en Île-de-France et des solidarités naturelles qui se nouent. En l'occurrence, 60 millions d'euros sont peu au regard des montants qui s'échangent quotidiennement dans la région.

Sans nier l'enjeu majeur que représente la production et ses mesures, la redistribution des richesses et leur valorisation sur le marché régional ne doivent pas être négligées. L'analyse que nous esquissons témoigne de l'importance de la consommation des ménages comme ressource économique pour les territoires et des effets de débordement qu'il peut exister. Des débordements qui cachent une inégale aptitude des territoires à satisfaire les attentes de leur population résidente et à capter le consommateur de passage. L'interdépendance des territoires est acquise mais l'intensité des échanges et des dissymétries varient considérablement.

Par nos résultats, nous soulevons la question du fonctionnement de l'économie métropolitaine, des équilibres, des spécialisations et des vulnérabilités qui la façonnent. Sur le sujet, il est difficile d'imaginer que les politiques publiques, notamment en matière de transport ou d'urbanisme commercial, n'aient aucune incidence sur l'organisation des marchés en Île-de-France. Depuis plusieurs années, les observateurs signalent un emballement de l'immobilier commercial en Île-de-France avec l'apparition de pôles commerciaux sans cesse plus grands et toujours plus concurrentiels. Or l'urbanisme commercial relève encore grandement de stratégies communales ou intercommunales. Des stratégies peu contrariées par les CDAC qui ont interdiction de statuer sur des considérations d'ordre économique. 
Accompagnées ou non, planifiées ou non, l'évolution de l'immobilier commercial et l'apparition de nouvelles formes de vente (click \& drive, vitrines éphémères...) sont de nature à restructurer l'organisation des marchés. La révolution commerciale d'aprèsguerre en témoigne encore. Dès lors, l'intensité et l'orientation des transferts de richesses observés vont-elles changer? Doit-on s'attendre à une évolution des rapports de force entre territoires? De quelle grille d'analyse disposerons-nous?

\section{Références}

Aguiléra A., Massot M-H., Proulhac L., 2010. Travailler et se déplacer au quotidien dans une métropole. Contraintes, ressources et arbitrages des actifs franciliens. Sociétés Contemporaines 80, 29-46.

Aguiléra A., Proulhac L., Wenglenski S., 2009. Employment Suburbanisation, Reverse Commuting and Travel Behavior by Residents of the Central City in the Paris Metropolitan Area. Transportation Research Part A: Policy and Practice 43 (7), 685-691.

André-Poyaud I., Chardonnel S., Charleux L., Tabaka K., 2006. La mobilité au cœur des emplois du temps des citadins In Chalas Y. (eds), La mobilité qui fait la ville, Certu, Lyon, 67-95.

Archer, B.H., 1996. Economic Impact Analysis. Annals of Tourism Research 23 (3), 704-707.

Ascher F., 2005. La société hypermoderne. Ces événements nous dépassent, feignons d'en être les organisateurs. Édition de l'Aube, La Tour d'Aigues.

Babayou P., Volatier J-L., 1998. Prospective de la mobilité locale (Collection des rapports. 186). CREDOC, Paris.

Bailly J.P., Heurgon E., 2001. Nouveaux rythmes urbains : quels transports? Édition de 1'Aube, La Tour d'Aigues.

Beaujeu-Garnier J., Chabot G., 1963. Traité de géographie urbaine. Armand Colin, Paris.

Beaujeu-Garnier J., Delobez A., 1977. Géographie du commerce. Masson, Paris.

Béhar D., Estèbe P., Vanier M., 2009. Mécano territorial : de l'ordre territorial à l'efficacité interterritoriale. Pouvoir locaux 83, 79-83.

Berry, B.-J.-L., 1971. Géographie des marchés et du commerce de détail. Armand Colin, Paris.

Bondue J-P. (dir.), 2004. Temps des courses, course des temps. USTL, Lille.

Bowles J., 2007. Recapturing Suburban Shoppers, Off the CUF. The Center for an Urban Future, New York.

Capon G. (dir.), 2011. L'évolution de la géographie du commerce de détail. Une approche par les déclarations annuelles de données sociales (Cahiers de recherche 285). CREDOC, Paris.

Chaze M., 2010. Du groupement des déplacements aux parcours quotidiens : la pérégrination du consommateur dans les petites villes françaises, In Boquet Y., Desse R-P. (eds), Commerce et mobilités. EUD, collections Sociétés, Dijon.

Constants-Martigny C., Ruault J-F., 2012. Urbanisme Commercial. Une implication croissante des communautés mais un cadre juridique à repenser. Études AdCF, juillet, Paris.

Cortright J., 2002. The Economic Importance of Being Different: Regional Variations in Tastes, Increasing Returns and the Dynamics of Development. Economic Development Quarterly 16, 3-16.

CRT Paris Île-de-France, 2011. Repères de l'activité touristique de la destination Paris Île-de-France 2011. Collection Repères 4.

Davezies L., 2007. Croissance sans développement en Île-de-France. Caisse des dépôts et consignations, Paris.

Davezies L., 2008. La République et ses territoires : la circulation invisible des richesses. Le Seuil, Paris.

Davezies L., 2010. Quels gisements de croissance dans les territoires? In Godet M., Créativité et Innovation dans les territoires. Conseil d'Analyse Économique, Paris.

Davezies L., 2011. Les agglomérations et leur territoire. Dix ans de dynamiques socio-économiques. Rapport préparé pour l'Association des Communautés de France, Paris. 
De la Court P., 1659. In Het Welvaren van Leiden (The Prosperity of Leiden). Leyde.

Delage M., 2011. Les équipements commerciaux d'Île-de-France, In Pumain D, Mattei M-F. (eds), Données Urbaines 6, Economica, Paris.

De la Mata T., Llano-Verduras C., 2011. Spatial Pattern and Domestic Tourism: An Econometric Analysis Using Inter-Regional Monetary Flows by Type of Journey. Papers in Regional Science 91 (2), 437-470.

Delaporte C., Courel J., 2006. Les déplacements pour achats. Analyse des comportements des franciliens en matière de déplacements pour achats. Les cahiers de l'enquête globale de transport 7, DREIF, juillet, Paris.

Delaporte C., 2012. Les enjeux métropolitains du commerce de l'agglomération de Paris. Panorama de l'organisation commerciale. APUR - IAU Île-de-France - Ville de Paris, juin, Paris.

Desse R-P., 2000. Les déterminants et les modes de mobilité des consommateurs. Bulletin de la société géographique de Liège 39 (2), 65-78.

Desse R-P., 2001. Le nouveau commerce urbain - dynamiques spatiales et stratégies des acteurs. PUR, Rennes.

Dissart J-C., Vollet D., 2010. Landscapes and Territory-Specific Economic Bases. Land Use Policy 28 (3), 563-573.

Engel E., 1857. Die Productions- und Consumtionsverhältnisse des Königreichs Sachsen. Statistischen Büreaus des Königlich Sächsischen Ministeriums des Innern, Dresden.

Erickcek G.A., Watts B.R., 2007. Market Gap Analysis for the Greater Grand Rapids Area. Report submitted to People and Land (PAL), March, Kalamazoo.

Farness D.H., 1989. Detecting the Economic Base : New Challenges. International Regional Science Review 12, 319-328.

Fletcher J.E., 1989. Input-Output Analysis and Tourism Impact Studies. Annals of Tourism Research 16, 514-29.

Fleury A., Mathian H., Saint-Julien T., 2012. Définir les centralités commerciales au cœur d'une grande métropole : le cas de Paris intra-muros. Cybergeo : European Journal of Geography, [URL] http://cybergeo. revues.org/25107.

Frank R-H., 2000. Luxury Fever. Money and happiness in an era of excess. Princeton University Press, Princeton.

Gallez C., Orfeuil J-P., 1998. Dis-moi où tu habites, je te dirai comment tu te déplaces, In Pumain D., Mattei M-F. (eds), Données urbaines 2, Anthropos, Paris.

Gasnier A., 2007. Les temps de mobilité des consommateurs au cœur des nouvelles logiques d'implantation de 1'offre commerciale. Espace Population Société 2-3, 243-254.

Glaeser E., Kolko J., Saiz A., 2001. Consumer city. Journal of Economic Geography 1, 27-50.

Gonnard S., 2001. Le procès de la base économique. Enquête sur l'interprétation, l'usage et la condamnation de la base économique par l'économie urbaine du XXe siècle. Mémoire de DEA, Institut d'Urbanisme de Paris, Université Paris-XII.

Greiner R., N. Stoeckl, R. Schweigert, 2004. Estimating Community Benefits from Tourism: The case of Carpentaria Shire. $48^{\text {th }}$ Annual Conference of the Australian Agricultural and Resource Economics Society, Melbourne, february 11-13.

Halbert L., 2004. The Decentralization of Intrametropolitan of Business Services in the Paris Region: Patterns, Interpretation, Consequences. Economic Geography 80 (4), 381-405.

Hermann R.O., Beik L.L., 1968. Shopper's Movement Outside their Local Retail Area. Journal of Marketing $32,45-51$.

IAURIF, 2013. Surproduction de surfaces commerciales, vers une bulle immobilière? Note Rapide 635, décembre.

Jarratt D.G., 2000. Outshopping Behaviour: an Explanation of Behaviour by Shopper Segment Using Structural Equation Modelling. International Review of Retail, Distribution and Consumer Research 10 (3), 287-304.

Krikelas A-C., 1992. Review of Economic Base Literature. In Economic Review, Federal Reserve Bank of Atlanta, July/August, 16-29. 
Kwon J., 2010. Import Substitution at the Regional Level: Application in the United States. Federal Reserve Bank of Atlanta.

Labasse J., 1955. Les Capitaux et la Région. Essai sur le commerce et la circulation des capitaux dans la région lyonnaise (Cahiers de la fondation nationale des sciences politiques 69). Armand Colin, Paris.

Lapeyronnie B., 2009. Retombées socio-économiques du tourisme sportif : exemples des marathons en France. Teoros 28 (2), 37-44.

Le Breton E., 2005. Bouger pour s'en sortir. Mobilité quotidienne et intégration sociale. Armand Colin, Paris.

Lejoux P., 2002. Esquisse d'une géographie des flux de revenu/consommation associés aux pratiques touristiques. Recherche pour le ministère du Tourisme/Direction du tourisme, L'Eil, Université Paris XII, Créteil.

Lemarchand N., 2009. Géographie du commerce et de la consommation, les territoires du commerce distractif. Mémoire d'habilitation à diriger la recherche, Université Paris IV.

Leo P-Y., Philippe J., 2000. Comportement des consommateurs dans l'espace métropolitain. Arbitrage entre centre-ville et périphérie commerciale. Actes de l'International Congress Marketing Trends.

Madry P., 2011. Le commerce est entré dans sa bulle. Études Foncières 151, 12-17.

Madry P., 2012. La fin de l'urbanisme commercial. Études Foncières 160, 20-24.

Mangin D., 2004. La ville franchisée. Formes et structures de la ville contemporaine Éd. de la Villette, Paris.

Massot M-H., Orfeuil J-P., 2005. La mobilité au quotidien, entre choix individuel et production sociale. Cahiers Internationaux de sociologie CXVIII, 81-100.

Massot M-H. (dir.), 2010. Mobilités et modes de vie métropolitains. Les intelligences du quotidien. Ed. 1'Oeil d'Or, Paris.

Markusen A., 2007. A consumption Base Theory of Development: An Application to the Rural Cultural Economy. Agricultural and Resource Economics Review 36 (1), 1-13.

Markusen A., Schrock G., 2009. Consumption-Driven Urban Development. Urban geography 30 (4), 344-367.

Maslow A.H., 1943. A Theory of Human Motivation. Psychological Review 50, 370-396.

Mérenne-Schoumaker B., 2008. Géographie des services et des commerces. PUR, Rennes.

Metton A. (dir.), 1984. Le commerce urbain français. PUF, Paris

Michaud-Trévinal A., Cliquet G., 2002. Localisation commerciale et mobilité du consommateur, Communication au $5^{\text {e }}$ colloque Étienne Thil, Paris, 26-27 septembre.

Moati P., Libouton J., Pouquet L., 2004. L'évolution de la géographie du commerce en France : une approche par les statistiques d'emploi (Cahier de recherche, CREDOC 207). CREDOC, Paris.

Moati P., 2011. La nouvelle révolution commerciale. Odile Jacob, Paris.

Moati P., 2012. Modes et lieux de consommation. Territoires 2040 - Des facteurs de changement 2, Datar, $27-41$.

Orfeuil J-P., 2010. La mobilité, nouvelle question sociale ? SociologieS, Dossiers, Frontières sociales, frontières culturelles, frontières techniques. http://sociologies.revues.org/3321.

Padeiro M., Pradel B., Aguiléra A., 2014. Paris sera toujours Paris : réflexions sur la centralité dans la métropole francilienne, Métropoles [URL] http://metropoles.revues.org/ - à paraître.

Papadopoulos N.G., 1980. Consumer Outshopping Research: Review and Extension. Journal of Retailing 56 (4), 41-58.

Péron, R., 2001. Le près et le proche. Les formes recomposées de la proximité commerciale. Les annales de la recherche urbaine $90,46-57$.

Péron, R., 2004. Les boîtes. Les grandes surfaces dans la ville. Librairie L'Atalante, Nantes.

Piketty, T., 2013. Le capital au XXI siècle. Le Seuil, Paris.

Rappaport, J., 2008. Consumption Amenities and City Population Density. Regional Science and Urban Economics 38, 533-552.

Rollier Y., Wiel M., 1993. La pérégrination. Mobilité et organisation de l'espace de Brest. AER/AUCUBE, CDU. 
Ruault J-F., 2012. Métropolisation et croissance de l'emploi de services aux ménages. Étude de cas de l'aire urbaine de Paris. Communication du Symposium Pour et Sur le Développement Régional (PSDR), Clermont-Ferrand, France, 19-21 juin.

Stynes D-J., 1997. Economic Impacts of Tourism. University of Illinois. Cooperative Extension Service bulletin, Champaign.

Tabaka K., 2009. Vers une nouvelle socio-géographie de la mobilité quotidienne. Étude des mobilités quotidiennes des habitants de la région urbaine de Grenoble. Thèse de doctorat de géographie, Université Joseph Fourier, Institut de Géographie Alpine de Grenoble, Grenoble.

Talandier M., 2013. Redéfinir l'enjeu de l'économie présentielle et le rôle des femmes dans les économies locales. Le cas des territoires de montagne. Journal of Alpine Research/Revue de géographie alpine 101-1.

Talandier M., Jousseaume V., 2013, Les équipements du quotidien en France : un facteur d'attractivité résidentielle et de développement pour les territoires? Norois 226, 7-23.

Talandier M., 2012. L'émergence de systèmes territoriaux productivo-résidentiels (SPR). Communication au XLIX $^{\mathrm{e}}$ colloque de l'ASRDLF, Belfort, 9-11 juin.

Talandier M., 2007. Un nouveau modèle de développement hors métropolisation : le cas du monde rural français. Thèse de Doctorat en urbanisme, aménagement et politiques publiques, Institut d'urbanisme de Paris, Créteil.

Terral L., Proulhac L., 2014. Les espaces de consommation : vers une économie résidentielle? In Guillaume R, Klein J-L (eds), Vers une nouvelle géographie économique, Presse de l'Université du Québec, Québec, 153-175.

Terrier C. (dir.), 2007. Mobilités touristiques et population présente. Les bases de l'économie présentielle des départements. Ministère des Transports, de l'Équipement, du Tourisme et de la Mer, Direction du Tourisme, Département Stratégie, Prospective, Évaluation et Statistiques.

Thévenin T., Chardonnel S. et Cochey E., 2007. Explorer les temporalités urbaines de l'agglomération de Dijon. Espace populations sociétés 2007 (2-3), 179-190.

Tiebout C-M., 1956. A pure Theory of Local Expenditures. Journal of Political Economy 64, 416-424.

Vacher L., Vye D, 2011. Fréquentation et dilatation de la ville moyenne touristique. Urbanisme 378, mai-juin.

Van de Walle I., 2005. Commerce et mobilité. L'activité commerciale face aux nouvelles politiques publiques de déplacements urbains (Cahier de Recherche, CREDOC 216). CREDOC, Paris.

Vanier, M., 2008. Le pouvoir des territoires. Essai sur l'interterritorialité. Economica, Paris.

Vollet D., 1998. Estimating the Direct and Indirect Impact of Residential and Recreational Functions on Rural Areas : an Application to Five Small Areas of France. European Review of Agricultural Economics 25 (4), 527-548.

Weber M., 1921. La ville. Aubier Montaigne, Paris.

Wesley S-C., LeHew M., 2002. Tourist-Oriented Shopping Centers: Investigating Customers' Evaluation of Attribute Importance. Journal of Shopping Center Research 9 (2), 31-52.

Williams C-C., 1997. Consumer Services and Economic Development. Routledge, London.

Zahavi Y., 1974. Travel Time Budgets and Mobility in Urban Areas. Report for the U.S. Department of Transportation, Washington, D.C. 